\title{
Transition Metal Synthetic Ferrimagnets: Tunable Media for All-Optical Switching Driven by Nanoscale Spin Current
}

Maciej Dąbrowski, ${ }^{, \dagger}$ Jade N. Scott, ${ }^{\ddagger}$ William R. Hendren, ${ }^{\ddagger}$ Colin M. Forbes, ${ }^{\ddagger}$ Andreas Frisk, ${ }^{\natural}$ David M. Burn, ${ }^{\complement}$ David G. Newman, ${ }^{\dagger}$ Connor R. J. Sait, ${ }^{\dagger}$ Paul S. Keatley, ${ }^{\dagger}$ Alpha T. N'Diaye,${ }^{\S}$ Thorsten Hesjedal, ${ }^{\|}$Gerrit van der Laan, ${ }^{\circledR}$ Robert M. Bowman, ${ }^{\ddagger}$ and Robert J. Hicken ${ }^{\dagger}$

${ }^{\dagger}$ Department of Physics and Astronomy, University of Exeter, Exeter, EX4 4QL, United Kingdom

${ }^{\ddagger}$ School of Mathematics and Physics, Queen's University Belfast, Belfast, BT7 1NN, United Kingdom

"Diamond Light Source, Harwell Science and Innovation Campus, Didcot, OX11 ODE, United Kingdom

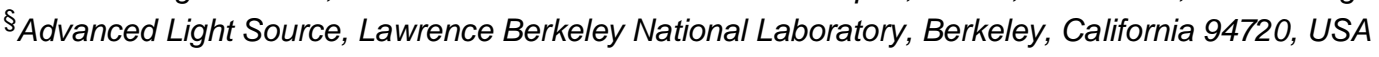

"Department of Physics, Clarendon Laboratory, University of Oxford, OX1 3PU, United Kingdom

\section{S1. Sample characterization}

In addition to the synthetic ferrimagnet ( $\mathrm{SFi}$ ) samples presented in the main text, reference $\mathrm{Co}$ and $\mathrm{Ni}_{3} \mathrm{Pt}$ samples with different thickness and deposition conditions were grown [1]. The thickness dependence of the Curie temperature $T_{\mathrm{C}}$ and atomic magnetic moment $\mu_{\mathrm{at}}$ for $\mathrm{Ni}_{3} \mathrm{Pt}$ obtained from SQUID measurements on reference $\mathrm{Ni}_{3} \mathrm{Pt}$ samples is shown in Fig. S1. For all SFi samples studied here, a Co layer with a fixed thickness of $1 \mathrm{~nm}$ was used, with $T_{\mathrm{C}}=$ $780 \mathrm{~K}$ and $\mu_{\mathrm{at}}=1.69 \mu_{\mathrm{B}}$ /atom [1]. The Ir spacer layer thickness was $0.5 \mathrm{~nm}$ for all the samples, and the thickness and growth conditions of the $\mathrm{Ni}_{3} \mathrm{Pt}$ layer were varied to tune the magnetic anisotropy and magnetization compensation point temperature $T_{\mathrm{M}}$ [1].
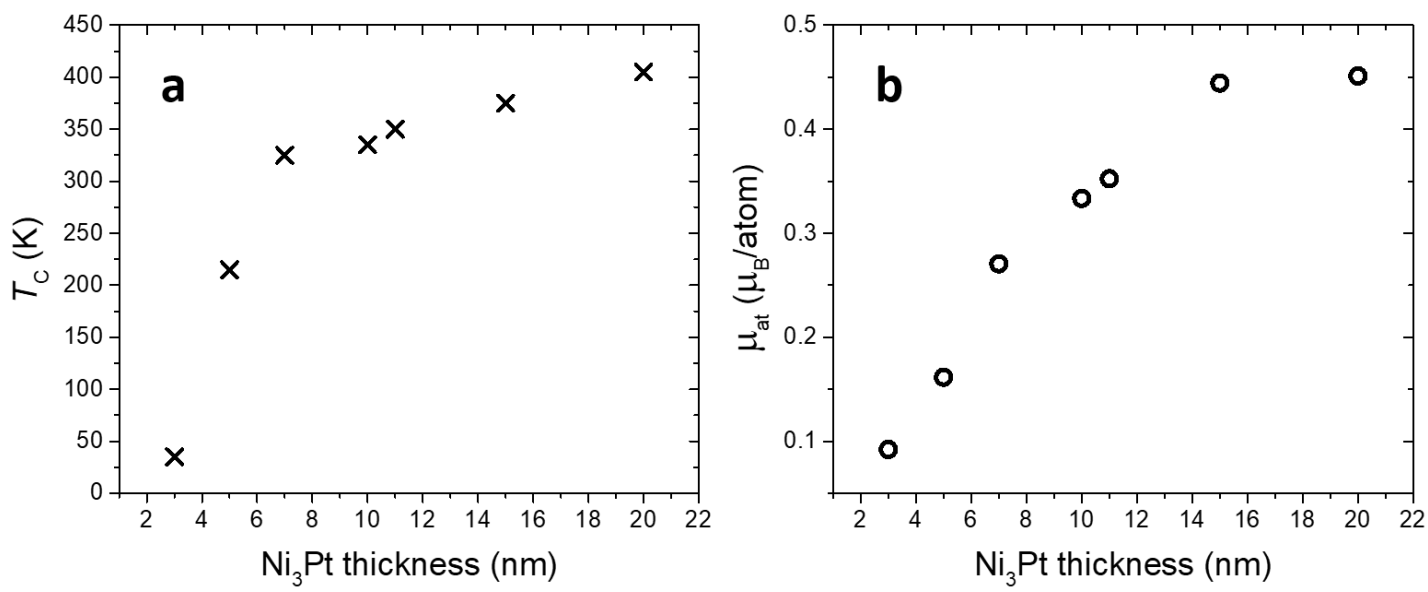

Fig. S1. Thickness dependence of the Curie temperature $T_{\mathrm{c}}(\mathrm{a})$ and atomic spin moment $\mu_{\mathrm{at}}(\mathrm{b})$ extracted from the saturation magnetization observed in SQUID measurements of $\mathrm{Ni}_{3} \mathrm{Pt}$ reference samples.

All samples labelled as $A, B, C, D$ and $E$ in the main text were characterized by SQUID magnetometry to determine the compensation temperature $T_{\mathrm{M}}$ and the negative remanence temperature range $\Delta T_{-\mathrm{R}}$ (as defined in Fig. 1a in the main text). The temperature dependent remanent magnetization $\mu_{0} M_{\mathrm{R}}$ (filled circles) extracted from the hysteresis loops measured at each $T$, and the remanent magnetization after applying the saturation field 
only at the beginning of the $T$ scan $\left|\mu_{0} M_{R}\right|$ (open squares), are plotted in Fig. S2 for samples A, D, E. This demonstrates that both $\Delta T_{-R}$ and $T_{M}$ can be tuned in the present system.

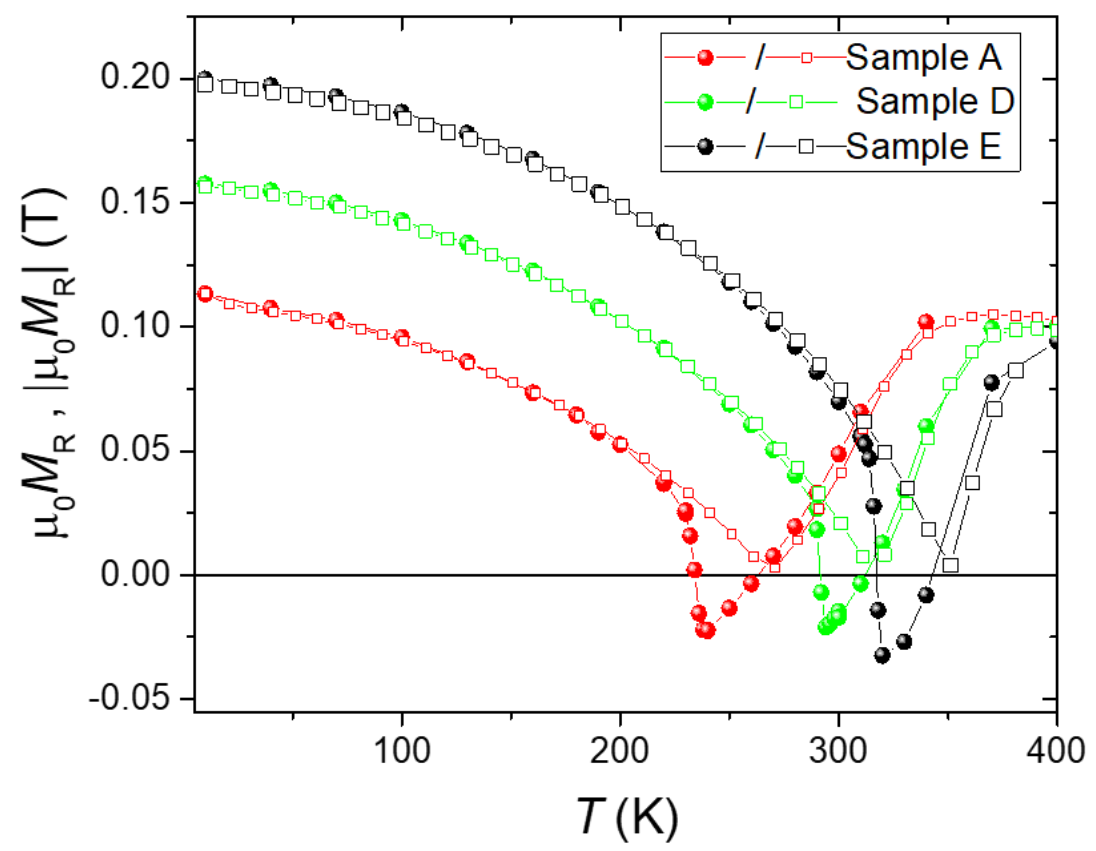

Fig. S2. Remanent magnetization as a function of temperature $T$ obtained from SQUID magnetometry, by measuring: i) the hysteresis loop at each $T$ ( $\mu_{0} M_{\mathrm{R}}$, filled circles); ii) the remanent magnetization after applying the saturation field only at the beginning of the $T$ scan $\left(\left|\mu_{0} M_{R}\right|\right.$, open squares) for three different samples $A, D$ and $E$, with the compensation points $T_{M}=271,310$ and $342 \mathrm{~K}$.

\section{S2. XMCD hysteresis loops and domain structure}

In Fig. S3 we show complementary results to Fig. 1d,e, with x-ray magnetic circular dichroism (XMCD) hysteresis loops for Sample $A$ at different temperatures. Below the transition into the negative remanence regime $\Delta T_{-R}, \mathrm{Ni}_{3} \mathrm{Pt}$ exhibits a normal square hysteresis loop, while Co exhibits negative coercivity ( $T=119$ and $217 \mathrm{~K}$ ). For temperatures at and above the transition to the $\Delta T_{-\mathrm{R}}$ regime, the behavior is opposite - $\mathrm{Ni}_{3} \mathrm{Pt}$ exhibits a loop with negative coercivity while the Co layer exhibits a normal hysteresis loop ( $T=227$, 232, and $268 \mathrm{~K})$. At $222 \mathrm{~K}$, a characteristic double-step hysteresis loop is observed, corresponding to the magnetization reversal exactly at the transition to the $\Delta T_{-\mathrm{R}}$ regime. This hysteresis loop can be explained by the superposition of XMCD signals from two or more regions that have either positive $(A P+)$ or negative remanence $(A P-)$, as shown schematically in Fig. S4. To verify this interpretation, we used wide-field Kerr microscopy (WFKM) to probe the magnetization reversal and domain structure the transition to the $\Delta T_{-R}$ regime. In the first step, we performed high quality (long averaging times) imaging at the magnetic field values corresponding to reversal of the $\mathrm{Ni}_{3} \mathrm{Pt}$ and Co layers. Below the $\Delta T_{-\mathrm{R}}$ regime, as we know from XMCD measurements (Fig. S3), the Co layer switches with negative coercivity, and the $\mathrm{Ni}_{3} \mathrm{Pt}$ with positive coercivity. Hence, while sweeping the field from $+100 \mathrm{mT}$, we can easily see that the magnetization reversal of the Co layer appearing 
at $+70 \mathrm{mT}$ proceeds via formation of large regular domains and domain wall motion (Fig. $\mathrm{S} 5 \mathrm{a})$. In contrast, the magnetization reversal of $\mathrm{Ni}_{3} \mathrm{Pt}$ occurring at $-76 \mathrm{mT}$ proceeds via dendritic domain pattern growth (Fig. S5b).

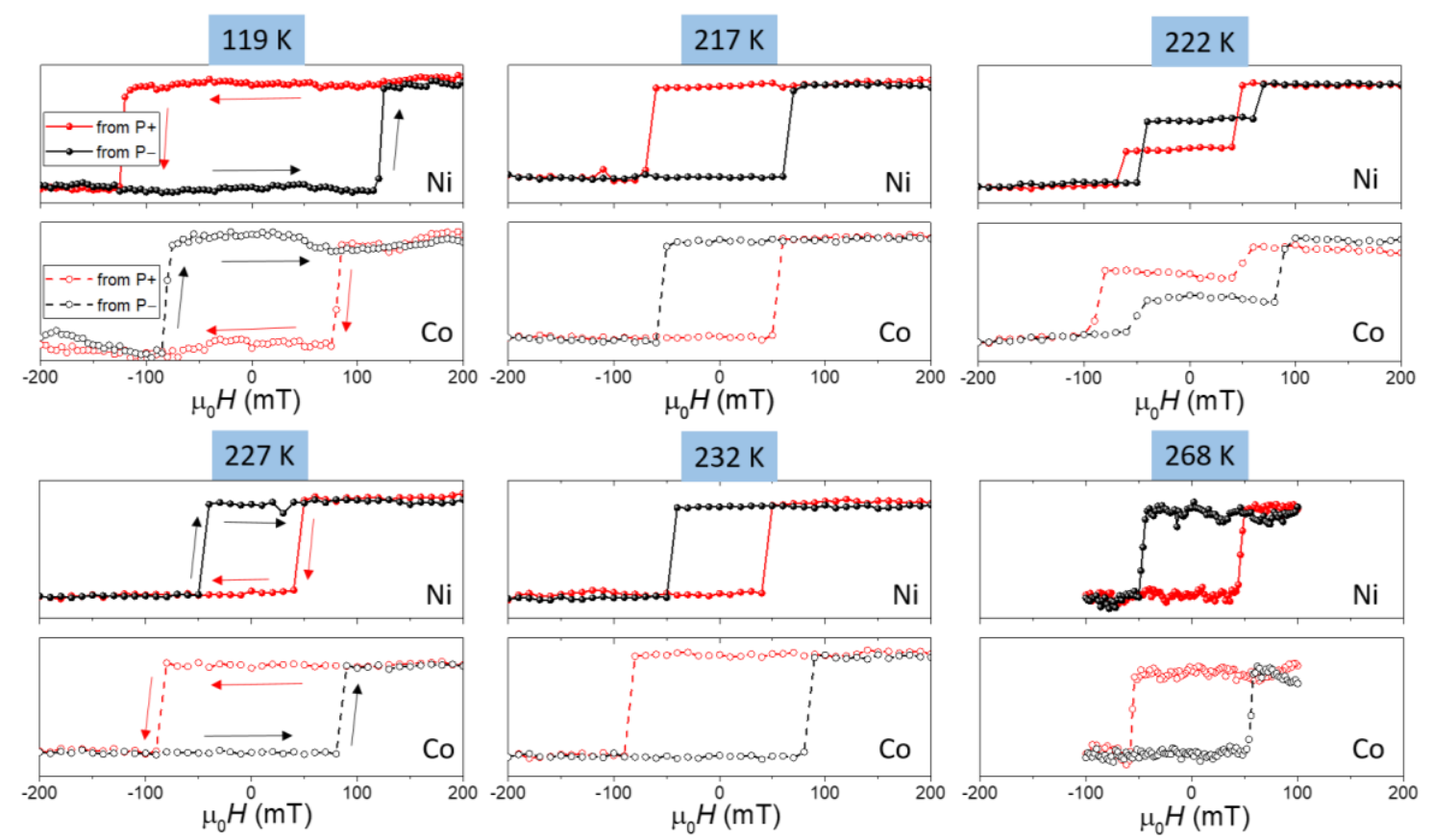

Fig. S3. XMCD hysteresis loops acquired at the $L_{3}$ absorption edges of $\mathrm{Ni}$ and $\mathrm{Co}$ at different temperatures for sample $\mathrm{A}\left[\mathrm{Ni}_{3} \mathrm{Pt}(8.5) / \mathrm{Ir}(0.5) / \mathrm{Co}(1)\right]$. Red and black data points correspond to the magnetic field sweep direction from positive saturation $(\mathrm{P}+)$ to negative saturation $(\mathrm{P}-)$, and from $\mathrm{P}-$ to $P+$, respectively.

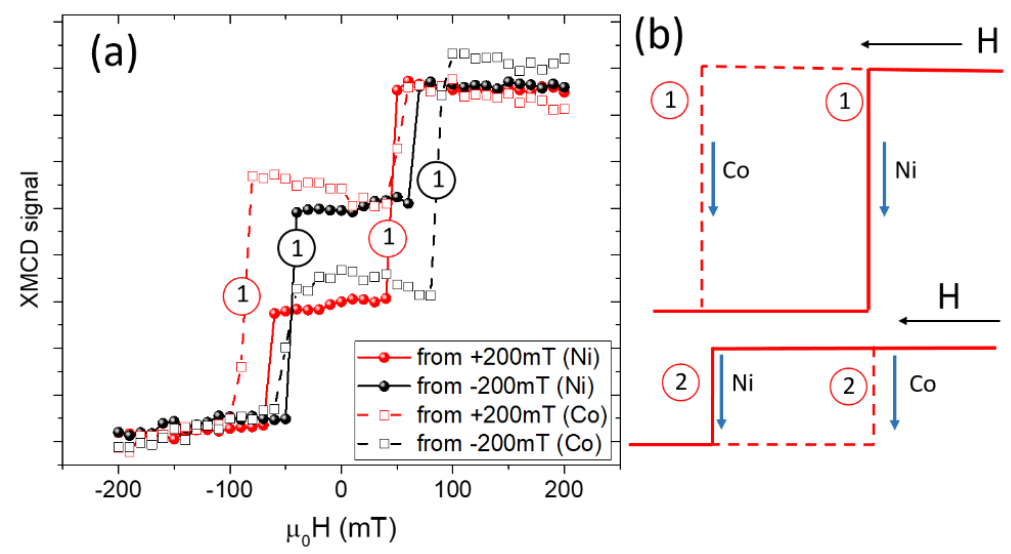

(c)

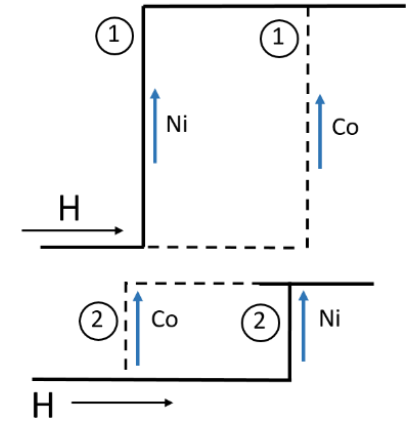

Fig. S4. (a) Double-step XMCD hysteresis loops obtained at the transition to negative remanence $T=$ $T_{-R}=222 \mathrm{~K}$ for $\mathrm{Ni}$ (filled circles/solid lines) and Co (open squares/dashed lines) for sample A. The hysteresis loops can be explained by the superposition of two different types of domains, with the $\mathrm{Ni}_{3} \mathrm{Pt}$ (Co) moments switching at negative (positive) coercive fields [marked schematically as (1)]; and the $\mathrm{Ni}_{3} \mathrm{Pt}$ (Co) moments switching at positive (negative) coercive fields [marked as (2)]. Schematic representation of signal contribution to the hysteresis loop from both types of domains for the magnetic field $H$ sweeping direction from positive to negative (b) and from negative to positive (c) values. 
The fact that the two layers exhibit different domain patterns during magnetization reversal is useful for identifying the reversal and orientation of a particular layer based solely on Kerr imaging. As expected from the XMCD loops, for temperatures above the transition to the $\Delta T_{-\mathrm{R}}$ regime, the sequence of domain patterns is interchanged, i.e., $\mathrm{Ni}_{3} \mathrm{Pt}$ switches at positive, and the Co at negative field, while sweeping the field from $+100 \mathrm{mT}$, as shown in Fig. S5e and Fig. S5f, respectively. By changing the temperature, we can capture the magnetization reversal right at the transition to the $\Delta T_{-\mathrm{R}}$ regime (i.e., $T=T_{-\mathrm{R}}$ ), where one can observe the coexistence of the two types of domains (Fig. S5c,d). In order to illustrate the magnetization reversal across the whole field range we show a sequence of Kerr images at different fields in Fig. S6. One can distinguish the coexistence of the two types of magnetization reversal while sweeping the field. The hysteresis loops reflecting the magnetization reversal for these two types of regions are plotted from the sample positions spot 1 and spot 2, as defined in Fig. S6. At spot 1, we observe positive remanence, while at spot 2 negative remanence is observed. While sweeping the field from positive saturation, the Co layers switch at positive (negative) field while the $\mathrm{Ni}_{3} \mathrm{Pt}$ at negative (positive) field at spot 1 (spot 2). The Kerr images therefore confirm the coexistence of two types of domains at the transition to the $\Delta T_{-\mathrm{R}}$ regime, and are consistent with a double-step XMCD loop (Fig. S4). Note that the coexistence of these two types of domains remains at remanence (see +0 $\mathrm{mT}$ in Fig. S6).
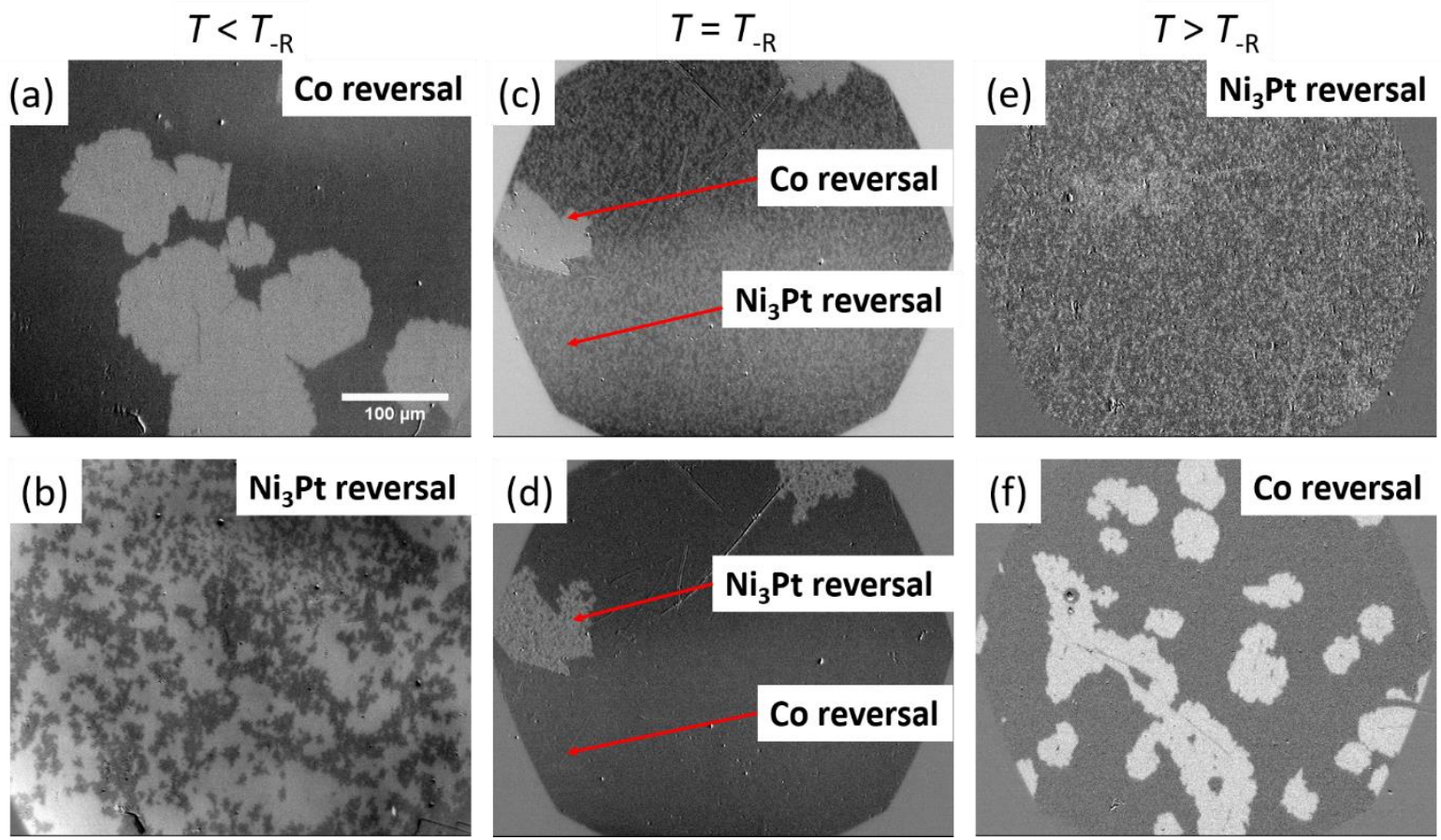

Fig. S5. Kerr microscopy images showing the reversal of the magnetization of the Co and $\mathrm{Ni}_{3} \mathrm{Pt}$ layers for sample A below $T_{-\mathrm{R}}$ at $T=190 \mathrm{~K}(\mathrm{a}, \mathrm{b})$; at $T_{-\mathrm{R}}$ where $T=241 \mathrm{~K}(\mathrm{c}, \mathrm{d})$ and above $T_{-\mathrm{R}}$ where $T=242 \mathrm{~K}$ $(e, f)$. The images were acquired while sweeping the field from positive saturation $+100 \mathrm{mT}$ at: (a) +70 mT -> (b) -76 mT; (c) +45 mT -> (d) -55 mT; (e) +44 mT -> (f) -68 mT. 
Finally, we would like to note that although the transition into the $\Delta T_{-\mathrm{R}}$ regime appears to be gradual when probed by SQUID magnetometry (Fig. 1a in the main text), it appears abrupt when probed by XMCD measurements and Kerr microscopy. This is because SQUID magnetometry spatially averages over the entire area of the sample, while XMCD and WFKM probe sub-millimeter sized local areas of the sample. Hence, the temperature range over which the coexistence of two types of reversal occurs is much wider when probed by SQUID magnetometry. Because the sample in not perfectly homogenous, and there are slight variations in the values of the magnetic parameters across the samples, the transition into the $\Delta T_{-\mathrm{R}}$ regime occurs at slightly different temperatures in different regions of the samples. With increasing temperature around the transition to the $\Delta T_{-\mathrm{R}}$ regime, SQUID measurements made at remanence therefore probe a gradual change in the population of the two antiparallel states $\mathrm{AP}+$ and $\mathrm{AP}-$, until all regions of the sample are in the negative remanence state. This corresponds to a negative extremum, as can be seen in Fig. 1a and Fig. S2. We also note that the uncertainty in the stated temperature values is $+/-5 \mathrm{~K}$, which is relevant when comparing different experimental techniques.
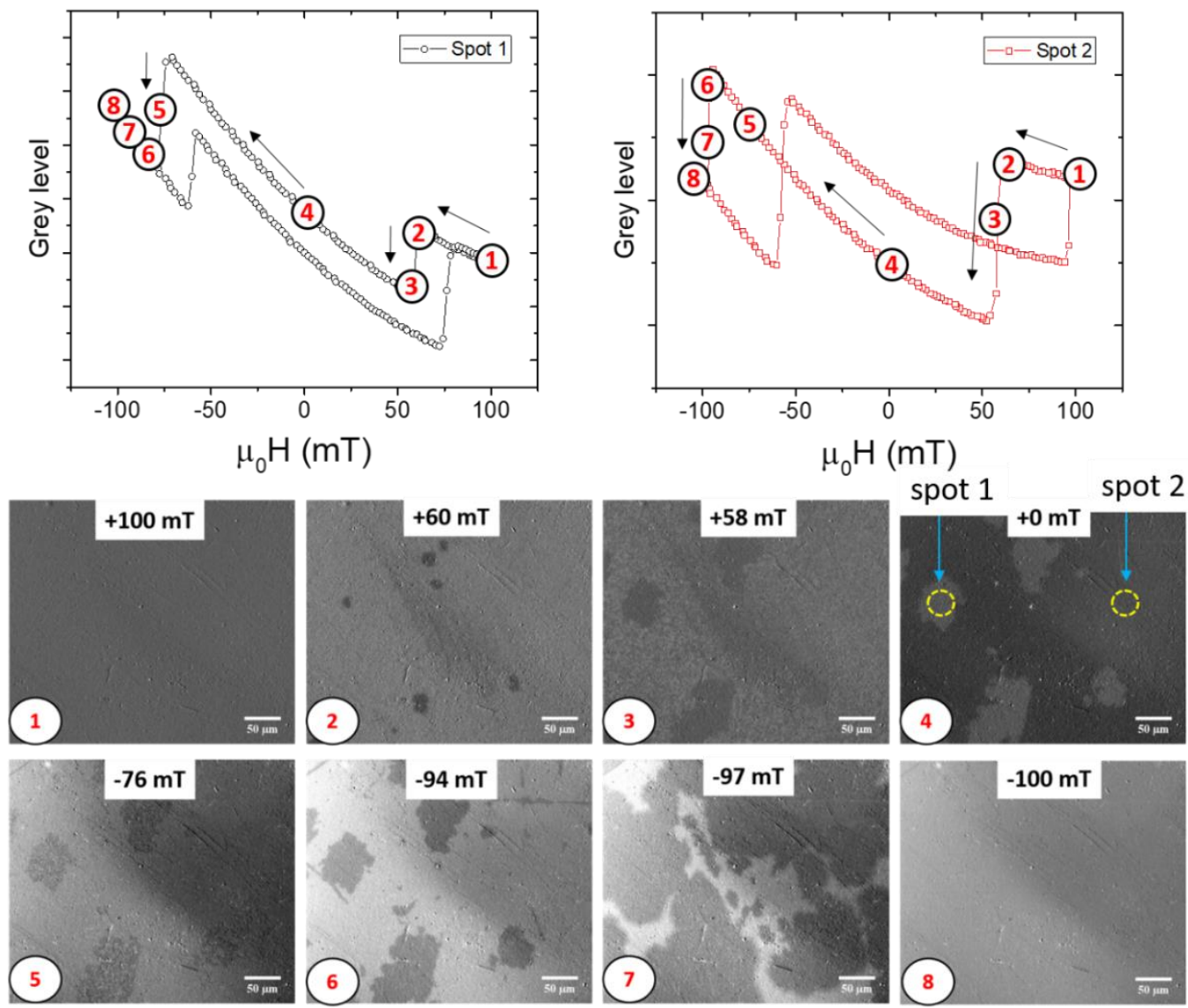

Fig. S6. Kerr microscopy images showing the magnetization reversal at the transition to the $\Delta T_{\text {-R }}$ regime for sample $A$ at $T=242 \mathrm{~K}$. The sequence of images shows two types of domains associated with reversal of $\mathrm{Co}$ and $\mathrm{Ni}_{3} \mathrm{Pt}$ while the magnetic field is swept from positive saturation field of +100 $\mathrm{mT}$ to negative saturation field of $-100 \mathrm{mT}$. By plotting the hysteresis loop from spot 1 and spot 2 , two different types of the magnetization reversal can be distinguished. At spot 1 , Co switches at positive field, while $\mathrm{Ni}_{3} \mathrm{Pt}$ switches at negative field, and hence this region is still in the 'positive' remanence state. In contrast, at spot $2, \mathrm{Ni}_{3} \mathrm{Pt}$ switches at positive field, while Co switches at negative field, and the region is in the negative remanence state. 
The nature of the transition into the $\Delta T_{-\mathrm{R}}$ regime is also reflected in the temperature dependence of the coercive fields $H_{C}$ of the two layers extracted from the XMCD loops, as shown in Fig. S7. The discontinuous jump in the coercive field observed at $222 \mathrm{~K}$ occurs exactly at the transition and is associated with the double-step hysteresis loop (Fig. S4). It is observed that the layer with the smaller coercivity (within the SFi hysteresis loop) always switches first as the field is decreased from saturation.

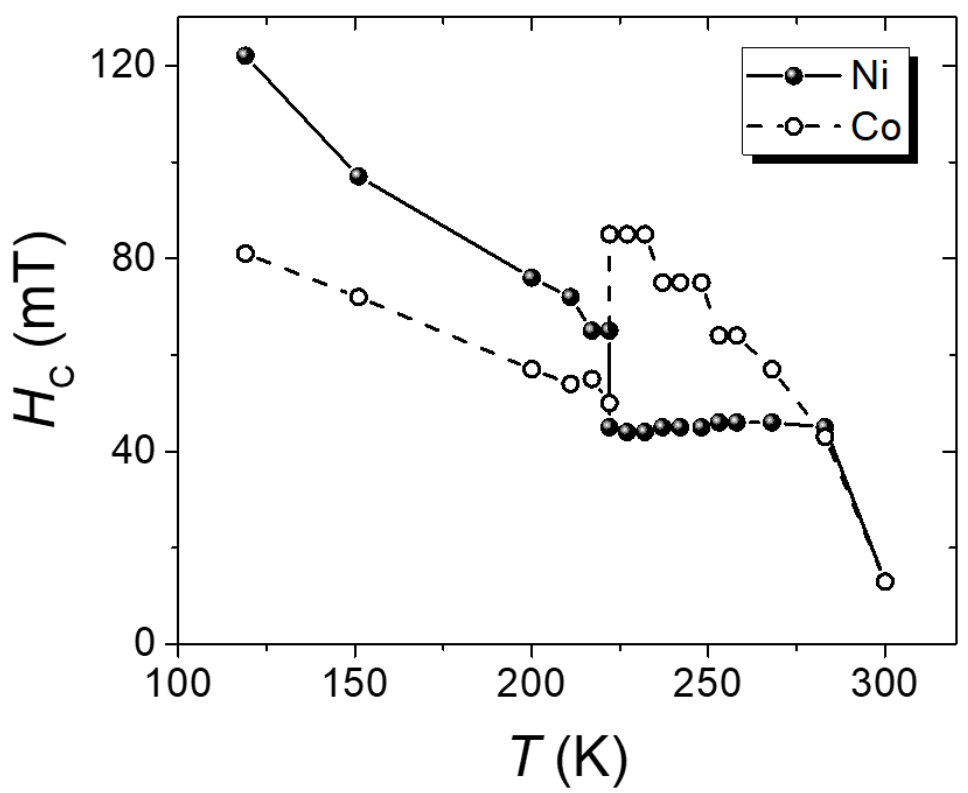

Fig. S7. Coercive field $H_{\mathrm{C}}$ as a function of temperature $T$ extracted from XMCD hysteresis loops for $\mathrm{Ni}$ (filled circles) and Co (empty circles) for sample A.

\section{S3. Optically induced domain structure creation and bias field assisted switching}

In Fig. S8 we show Kerr images demonstrating domain creation/demagnetization upon optical pumping for two different initial remanent state AP- and AP+ using different polarizations and different numbers of pulses. A random distribution of domains is observed with the two remanent states occurring with equal probability. Because the optical pump is incident at $45^{\circ}$ with respect to the normal direction, the resulting multi-domain pattern has an elliptical shape, and its size depends upon the polarization, i.e., it is slightly different for circularly and linearly polarized light because p-polarized light is absorbed more strongly than s-polarized light at grazing incidence. Exposure to a larger number of pulses results in similar domain patterns, with slightly extended perimeters and with slightly larger domain size (Fig. S8d-f). Note that pumping conditions such as those in Fig. S8f can already result in a permanent change in the magnetic properties of the sample, which is reflected in a slightly modified coercive field for the exposed region. Regardless of the number of pulses, the pump helicity does not seem to affect the final state. 
Note that images are presented in Fig. S8 after a background subtraction (BS). The BS process equalizes the grey scale of the initial state, as shown in Fig. S8g. For most of the images presented in this work (also in Fig. 2 of the main text), raw images (without BS) were used, to simplify the color scaling and interpretation of the intensity changes due to optical pumping. In particular, raw images allow one to immediately scale the contrast changes by 'eye', by positioning the pump beam next to an oppositely oriented remanent state, as shown in Fig. S9.
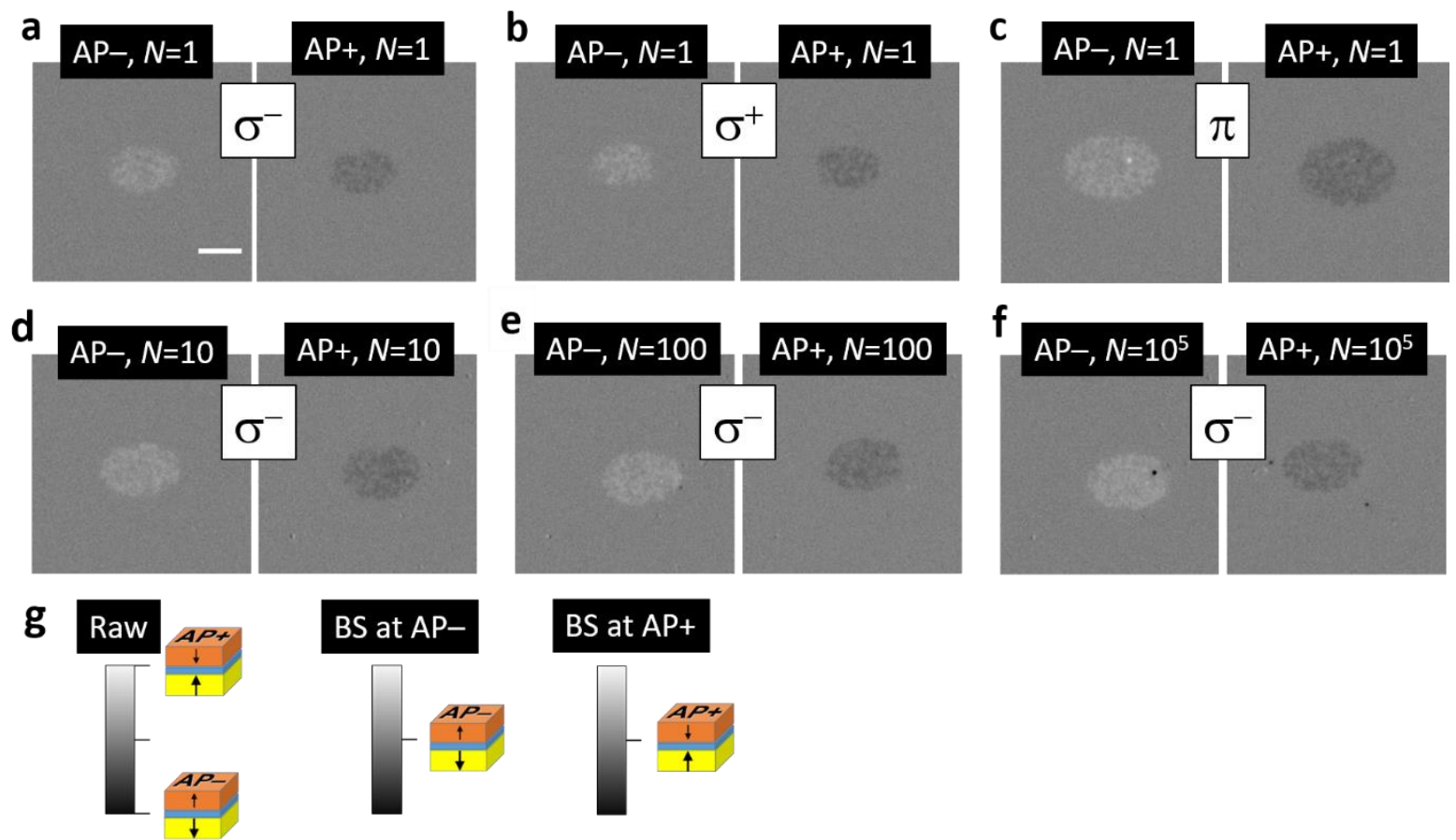

Fig. S8. Optically induced domain formation/demagnetization. (a-c) Kerr microscopy images obtained after exposure to a single laser pulse $(N=1)$ for two remanent initial states AP- and AP+, and for different polarizations of the laser pulse: left circular $\left(\sigma^{-}\right)$, right circular $\left(\sigma^{+}\right)$and linear p-pol $(\pi)$. (d-f) Effect of different numbers of laser pulses $N=10,100$ and $10^{5}$ with $\sigma^{-}$polarization. The initial state was reset after every measurement. All images were acquired with laser fluence $F=15$ $\mathrm{mJ} / \mathrm{cm}^{2}$, pulse duration $P D=1 \mathrm{ps}$, and $1 \mathrm{MHz}$ repetition rate. All measurements were performed at RT for Sample E [( $\left.\mathrm{Ni}_{3} \mathrm{Pt}(11) / \operatorname{Ir}(0.5) / \mathrm{Co}(1)\right]$. (g) Process of background subtraction (BS). AP+ and APstates correspond to different grey level on a raw image color scale. The BS process resets the grey scale so the AP+ and AP- initial states start from the same intensity level. 


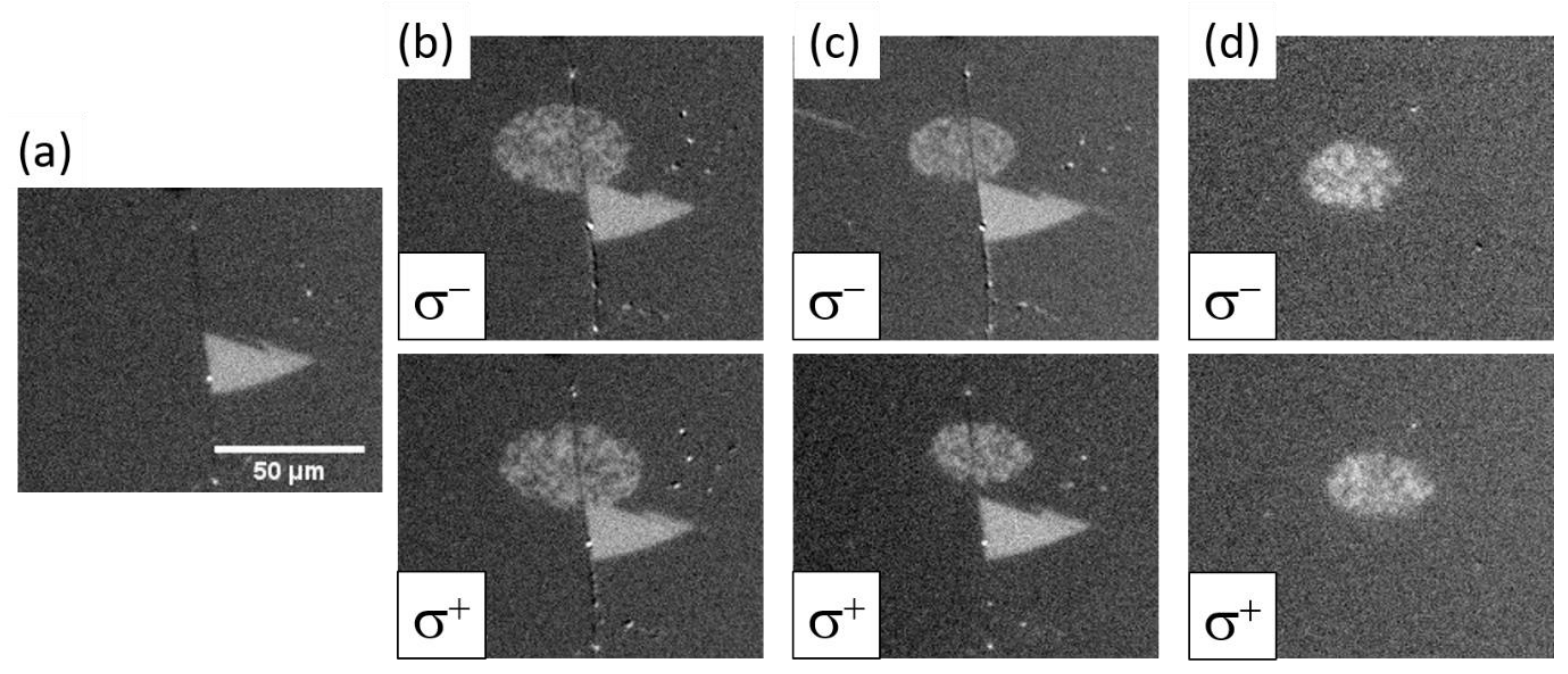

Fig. S9. Optically induced domain formation for two pump circular polarizations $\sigma^{-}$and $\sigma^{+}$, and for different laser pump parameters: (b) pulse duration $(P D)=0.5 \mathrm{ps}$, number of pulses $(N)=10^{4}$ at 100 $\mathrm{kHz}$ repetition rate; (c) $P D=1.5 \mathrm{ps}, N=100$ at $100 \mathrm{kHz}$ repetition rate and (d) $P D=1 \mathrm{ps}, N=10^{4}$ at 10 $\mathrm{kHz}$ repetition rate. (a) Shows the initial state, before pumping in (b). The initial state was reset after every measurement. In all the images $F=15 \mathrm{~mJ} / \mathrm{cm}^{2}$. Measurements were performed at RT for sample $\mathrm{E}\left[\mathrm{Ni}_{3} \mathrm{Pt}(11) / \mathrm{Ir}(0.5) / \mathrm{Co}(1)\right]$.

As discussed in Note 2 of the Supplementary Information, the coexistence of two states AP+ and AP- is possible at the transition to the $\Delta T_{-R}$ regime. In Fig. S9a the initial state was achieved by reaching remanence from negative saturation $\mathrm{P}$-. The darker domain corresponds to the AP-state, while a triangular shaped domain is in the AP+ state. Hence, the brighter AP+ domain is already in the negative remanent state. Figure S9 shows the effect of pumping with the circular polarizations $\sigma^{-}$and $\sigma^{+}$, and for different pulse duration (PD) and repetition rates. For all pumping conditions presented, optically induced domain creation is independent of the helicity of the laser pump, and no evidence of magnetic circular dichroism (MCD) affecting the final state is observed, similar to what was observed in Fig. S8. The optically created domains are composed of randomly distributed domains corresponding to the AP- (darker) and AP+ (brighter) remanent state.

In Fig. S10, we show the effect of a single optical pump pulse with a bias field applied normal to the sample for the initial remanent state AP+. It is clear that switching can be either promoted (positive fields) or hindered (negative fields) for the magnetic field oriented antiparallel or parallel to the magnetization of the Co layer, respectively. This demonstrates, that the final ordering of the ferrimagnet is dictated by the Co layer (FM layer with higher $T_{c}$ ). Full optical switching can be achieved at a field of $+3 \mathrm{mT}$ for this particular sample. For multi-pulse excitation, full optical switching can be observed for fields as low as $0.5 \mathrm{mT}$. 


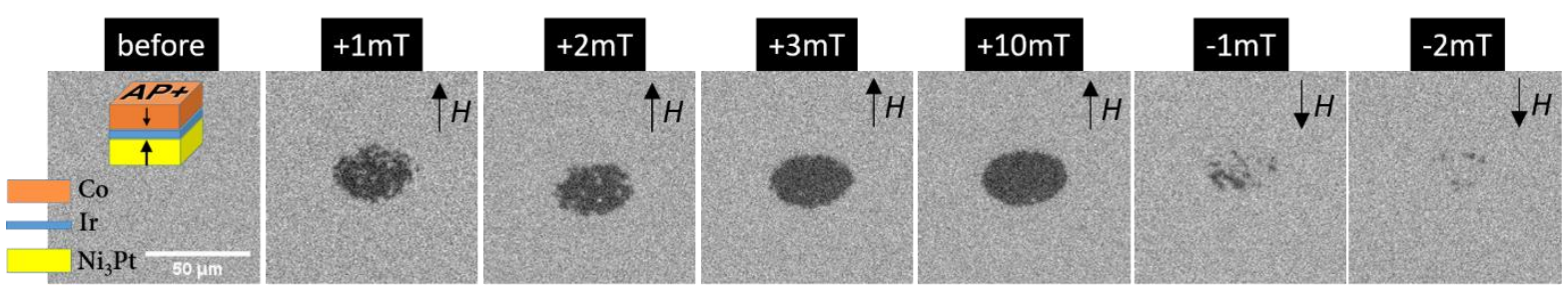

Fig. S10. Magnetic field assisted switching with a single laser pulse $(N=1)$ and linear polarization $\pi$, fluence $F=10 \mathrm{~mJ} / \mathrm{cm}^{2}$ and $P D=25 \mathrm{fs}$. The initial state of the sample was prepared by sweeping the field from positive saturation field to AP+. The optical pumping was assisted by either a positive field, i.e., aligned antiparallel (parallel) to $\mathrm{Co}\left(\mathrm{Ni}_{3} \mathrm{Pt}\right)$ magnetization direction, or a negative field, i.e., aligned parallel (antiparallel) to the $\mathrm{Co}\left(\mathrm{Ni}_{3} \mathrm{Pt}\right)$ magnetization direction. The initial state was reset after every measurement, and the images are background subtracted as described in Fig. S8g. The measurements were performed at $T=286 \mathrm{~K}$ (below $T_{-\mathrm{R}}$ ) for sample $\mathrm{D}\left[\mathrm{Ni}_{3} \mathrm{Pt}(10) / \mathrm{Ir}(0.5) / \mathrm{Co}(1)\right]$.

\section{S4. All optical switching (AOS)}

We now present AOS results complementary to those in the main article. In Fig. S11 we show AOS for a different number of pulses $(N)$. The laser beam is positioned as indicated by the yellow dashed ellipse, next to an oppositely oriented domain in the AP+ state. By increasing $N$, the size of the region switched from the AP- to the AP+ state is increased.
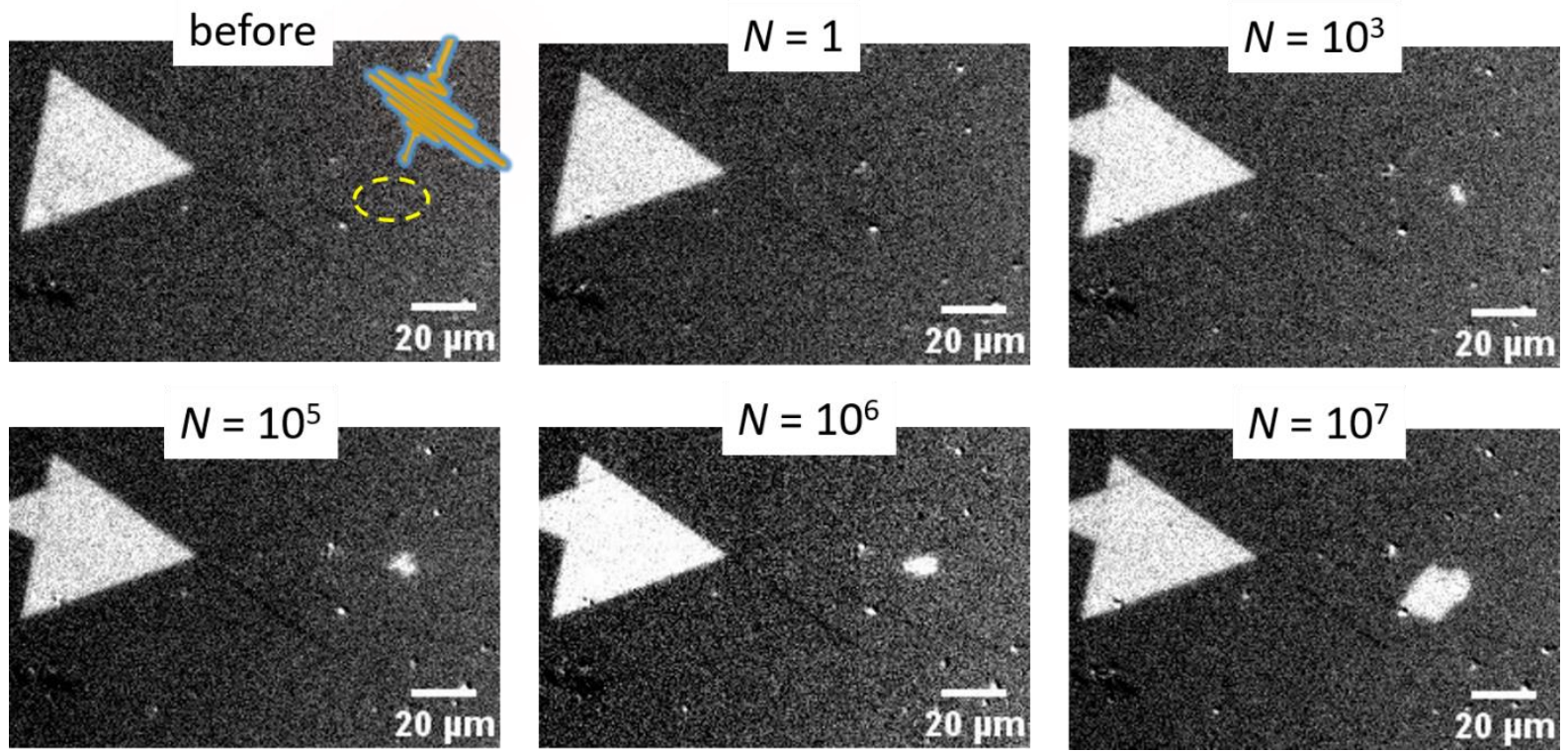

Fig. S11. All optical switching for different number of pulses $N, \sigma^{-}$polarization, pulse duration $P D=0.5 \mathrm{ps}$ at $1 \mathrm{MHz}$ repetition rate and fluence $F=10 \mathrm{~mJ} / \mathrm{cm}^{2}$. The initial state was reset to the APremanent state after every measurement. The measurements were performed at RT for sample $E$ $\left[\mathrm{Ni}_{3} \mathrm{Pt}(11) / \operatorname{Ir}(0.5) / \mathrm{Co}(1)\right]$. 
In Figs. S12-S14 we show AOS for sample $B$, induced with fluence $F=10 \mathrm{~mJ} / \mathrm{cm}^{2}, \sigma^{-}$ polarization, $P D=1 \mathrm{ps}$, and different number of pulses $N$ at $1 \mathrm{MHz}$ repetition rate. Here we do not reset the initial state, to verify whether toggle switching is possible. For $N=10^{3}$ pulses, the switched areas are nearly uniform but once switched, cannot be switched back. For $N=10^{4}$ pulses, the switching areas are uniform, but again, with no toggle switching. For $N=10^{5}$ pulses (Fig. S13), the switching areas are uniform and toggle switching is observed, i.e., the remanent state is switched back and forth without resetting the initial state. Toggle switching is achieved without changing the helicity and hence helicity independent AOS (HIAOS) is observed here. By increasing the number of pulses even further, to $N=10^{6}$ (Fig. S14), the AOS is uniform but the toggle switching is inconsistent and appears to be more random. Finally, in Fig. S15, optical pumping is performed with the same laser parameters as in Fig. S14 but for shorter pulse durations $P D=276$ and $500 \mathrm{fs}$, which leads to demagnetization/domain formation, while no AOS can be observed. We find that uniform switching can only be achieved with reduced laser fluence (peak power), where the centre of the Gaussian beam intensity profile leads to a monodomain state. Exposure to shorter $P D$ in Fig. S15 leads to a multidomain state because the peak power is larger than that used in Fig. S12-S14. However, other effects of the extended PD on the switching mechanism cannot be excluded.

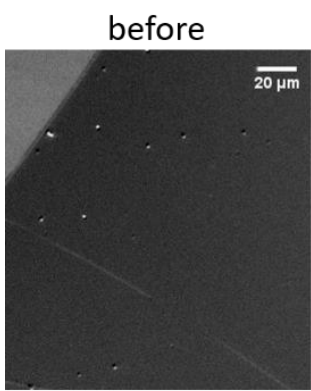

before

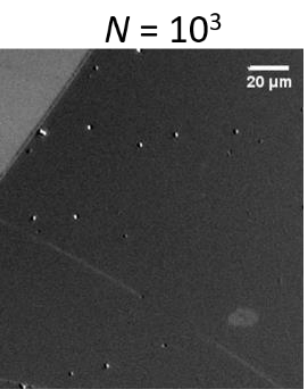

$N=10^{4}$

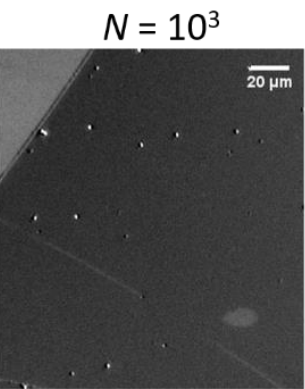

$N=10^{4}$

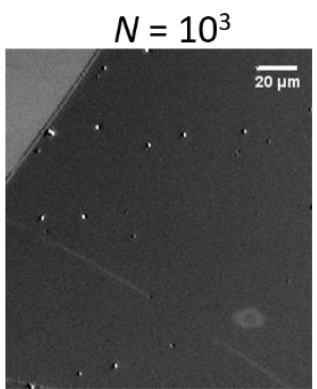

$N=10^{4}$

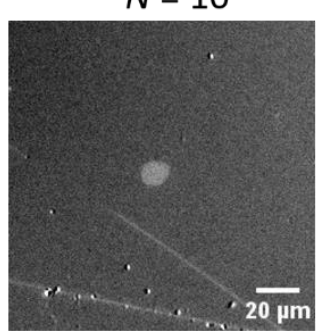

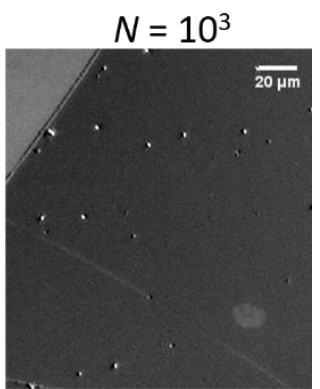

$N=10^{4}$
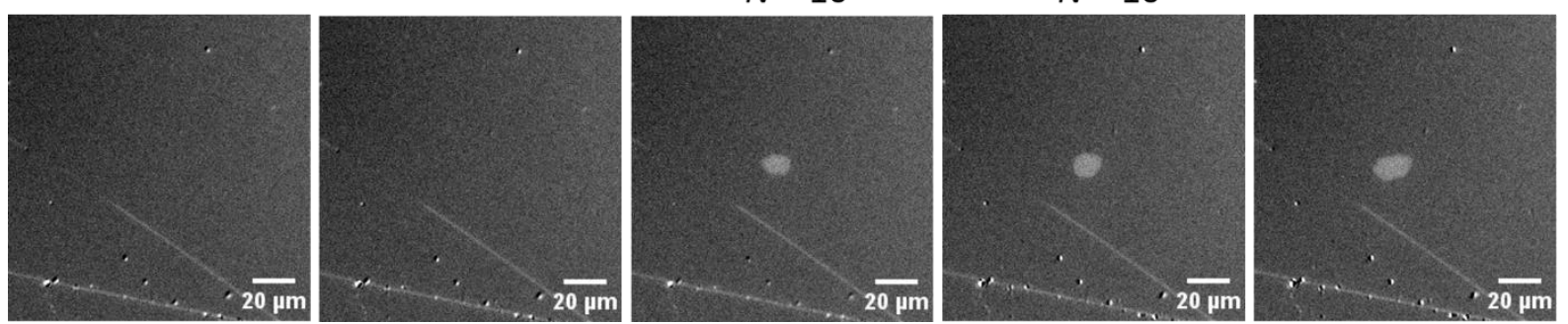

Fig. S12. All optical switching for number of pulses $N=10^{3}$ and $10^{4}, \sigma^{-}$polarization, pulse duration $P D$ $=1 \mathrm{ps}, 1 \mathrm{MHz}$ repetition rate and fluence $F=10 \mathrm{~mJ} / \mathrm{cm}^{2}$. Moving from left to right, each panel receives another $N$ pulses, without resetting the initial state. Measurements were performed at $T=230 \mathrm{~K}$ for sample B $\left[\mathrm{Ni}_{3} \mathrm{Pt}(8.5) / \mathrm{Ir}(0.5) / \mathrm{Co}(1)\right]$. 

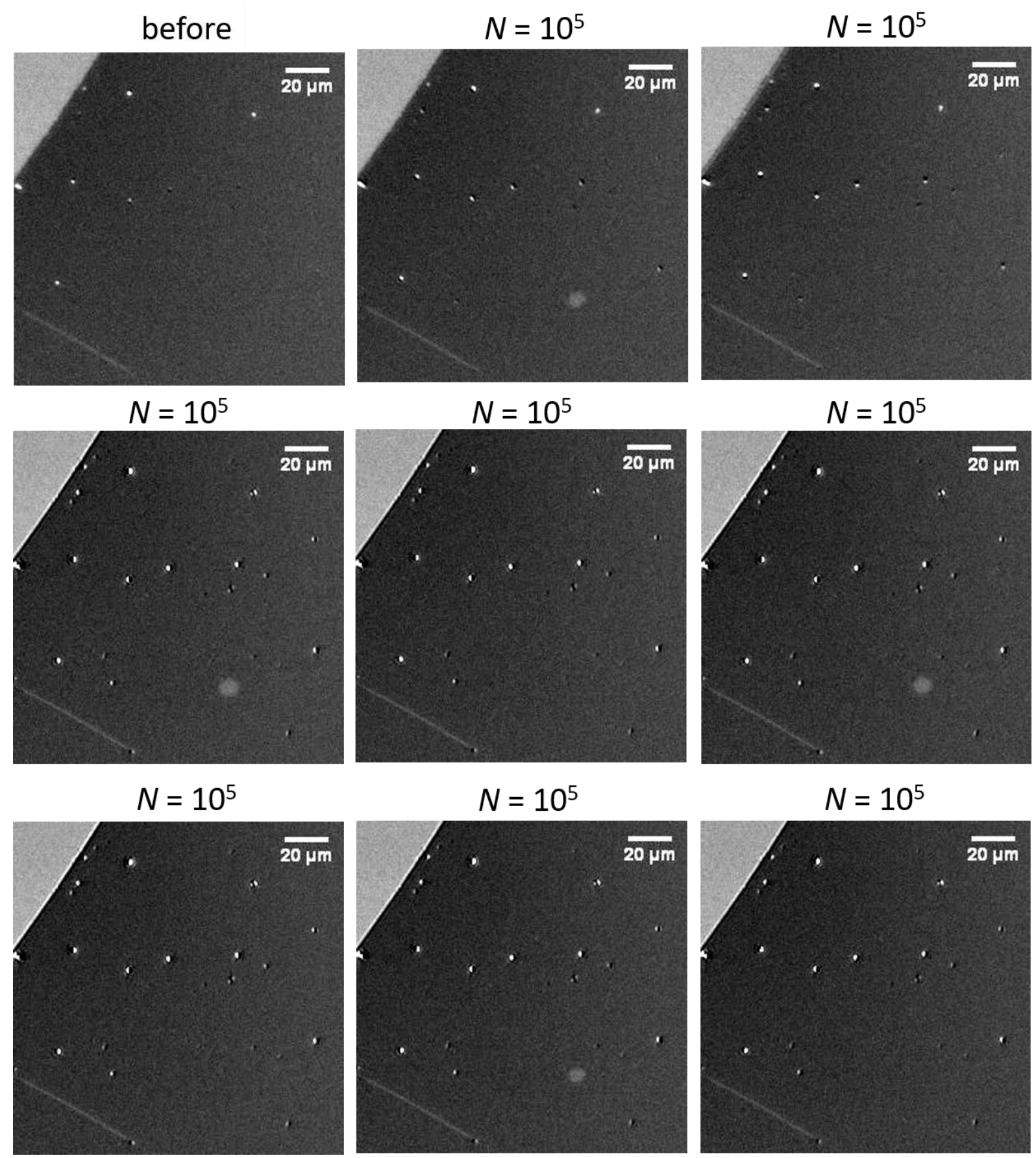

Fig. S13. Observation of all optical toggle switching for $N=10^{5}$ number of pulses, $\sigma^{-}$polarization, pulse duration $P D=1 \mathrm{ps}, 1 \mathrm{MHz}$ repetition rate and fluence $F=10 \mathrm{~mJ} / \mathrm{cm}^{2}$. Moving from left to right, each panel receives another $N$ pulses, without resetting the initial state. Measurements were performed at $T=230 \mathrm{~K}$ for sample B. 

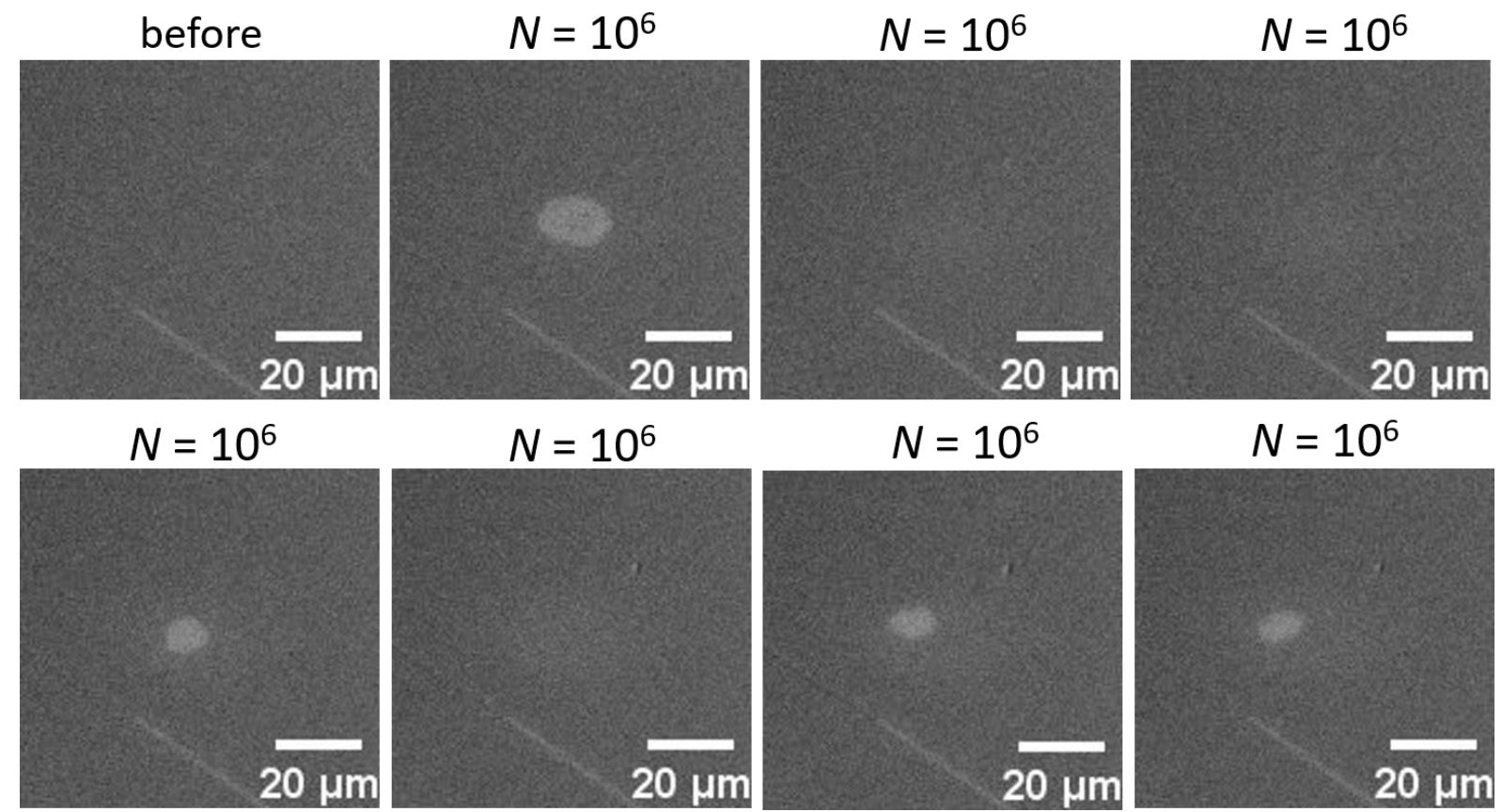

Fig. S14. All optical switching for $N=10^{6}$ pulses, $\sigma^{-}$polarization, $P D=1 \mathrm{ps}, 1 \mathrm{MHz}$ repetition rate and $F=10 \mathrm{~mJ} / \mathrm{cm}^{2}$. Moving from left to right, each panel receives another $N$ pulses, without resetting the initial state. Measurements were performed at $T=230 \mathrm{~K}$ for sample B.

$$
P D=275 \mathrm{fs} ; N=10^{6}
$$
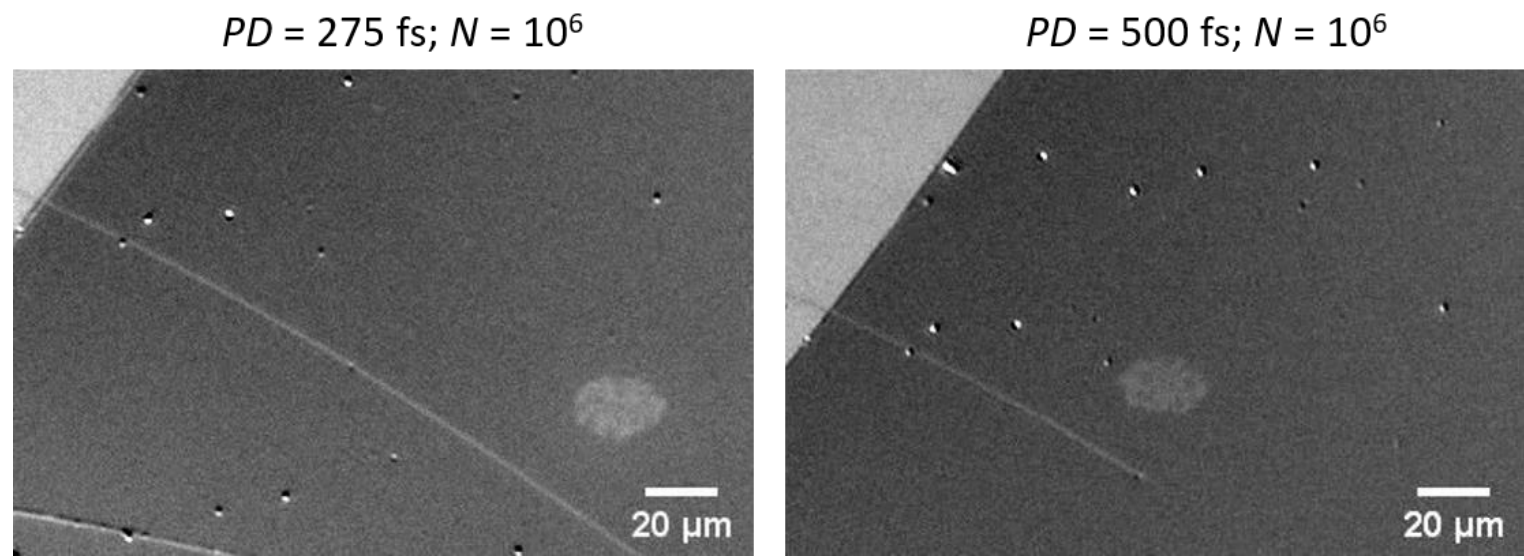

Fig. S15. Optically induced domain formation for different $P D=275 \mathrm{fs}$ and $500 \mathrm{fs}, N=10^{6}$ pulses, $\sigma^{-}$ polarization, $1 \mathrm{MHz}$ repetition rate and $F=10 \mathrm{~mJ} / \mathrm{cm}^{2}$. Measurements were performed at $T=230 \mathrm{~K}$ for sample B.

Similar demagnetization/domain formation and dependence of AOS upon the pulse duration can be observed for other samples, as shown for sample $B$ in Fig. S16. For $P D=$ $0.275,1$ and $1.5 \mathrm{ps}$, a multidomain state is recorded. For longer pulse duration $P D=3 \mathrm{ps,}$ uniform AOS is clearly visible, and more importantly, toggle switching can be achieved (Fig. S16c). 
(a)

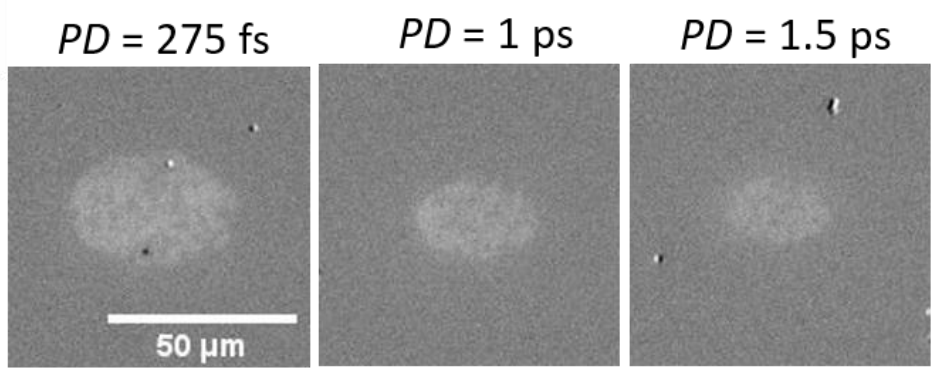

(b)
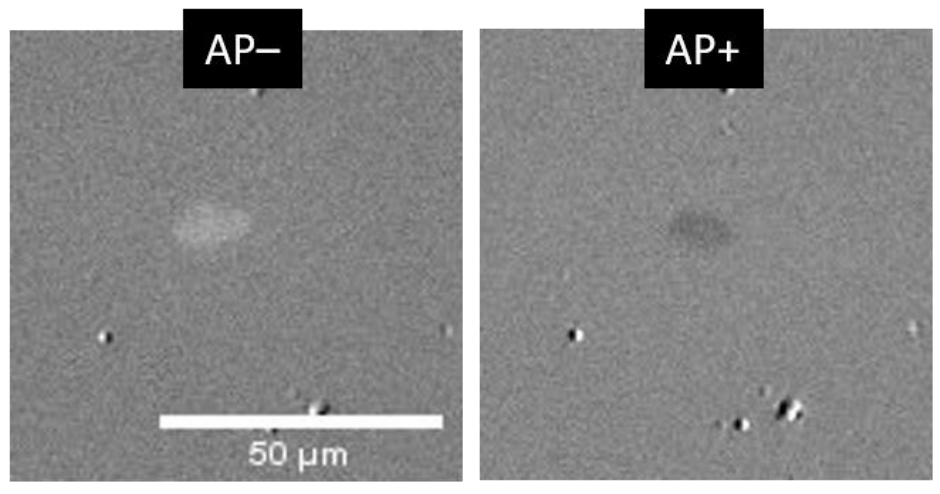

(c)
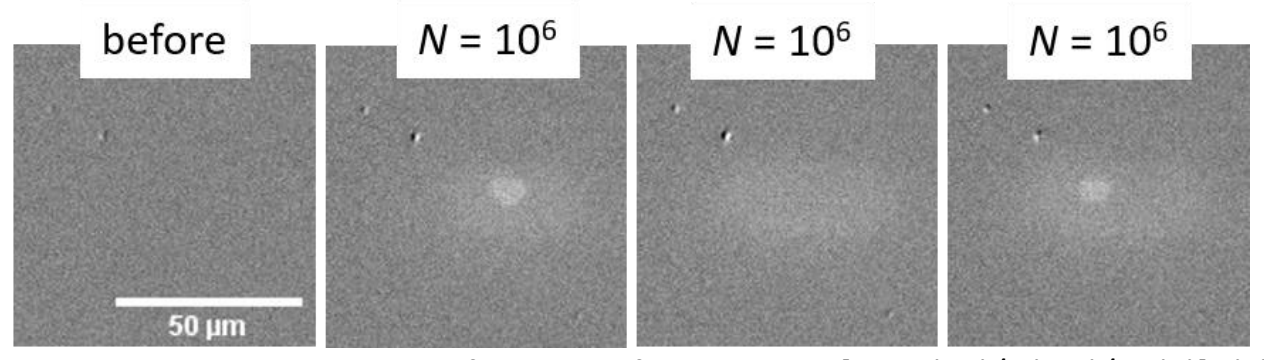

Fig. S16. All optical switching and domain formation for sample C [ $\left.\mathrm{Ni}_{3} \mathrm{Pt}(10) / \operatorname{Ir}(0.5) / \mathrm{Co}(1)\right]$. (a) Effect of different pulse duration $P D$. (b) AOS for different initial remanent states with $P D=3 \mathrm{ps}$. (c) toggle switching with $P D=3 \mathrm{ps}$. The measurements were performed at $T=280 \mathrm{~K}$, with number of pulses $N$ $=10^{6}, \sigma^{-}$polarization, $1 \mathrm{MHz}$ repetition rate and fluence $F=13 \mathrm{~mJ} / \mathrm{cm}^{2}$.

\section{S5. AOS with the beam swept along the sample surface}

We recorded Kerr images after sweeping the pump laser beam in a straight line. For a beam with pulse repetition rate of $1 \mathrm{MHz}(100 \mathrm{kHz})$ moving at a speed of $100 \mu \mathrm{m} / \mathrm{s}$, every point on this line receives nearly $10^{6}\left(10^{5}\right)$ pump pulses. We performed this experiment for samples with different $\mathrm{Ni}_{3} \mathrm{Pt}$ thicknesses (different compensation points $T_{\mathrm{M}}$ ) for varying sample temperature and fluence. In Fig. S17 and Fig. S18 we present the effect of different temperature and pump helicity on the AOS with the beam swept for sample $F$ $\mathrm{Ni}_{3} \mathrm{Pt}(10) / \mathrm{Ir}(0.5) / \mathrm{Co}(1) / \mathrm{Ir}(3)\left(T_{\mathrm{M}}=295 \mathrm{~K}\right)$. At $268 \mathrm{~K}$, we approach the transition to negative remanence state $T_{-\mathrm{R}}$ and hence we can observe domains with oppositely oriented remanent state. In Fig. S18a we show that domains in the negative remanence state (see large bright triangular domain) cannot be switched. Images taken after exposure to two different helicities confirm the HI-AOS. In Fig. S19 we show AOS for sample D for different fluences and for different laser pump polarizations, which again confirms HI-AOS. In Fig. S2O we present demagnetization and optically induced domain creation for reference ferromagnetic 
samples. Figure S21 shows the occurrence of AOS for sample $A$ and $F$ as a function of temperature $T$ and fluence $F$. AOS is seen for a wide range of fluence, but only for $T<T_{\text {-R. }}$.
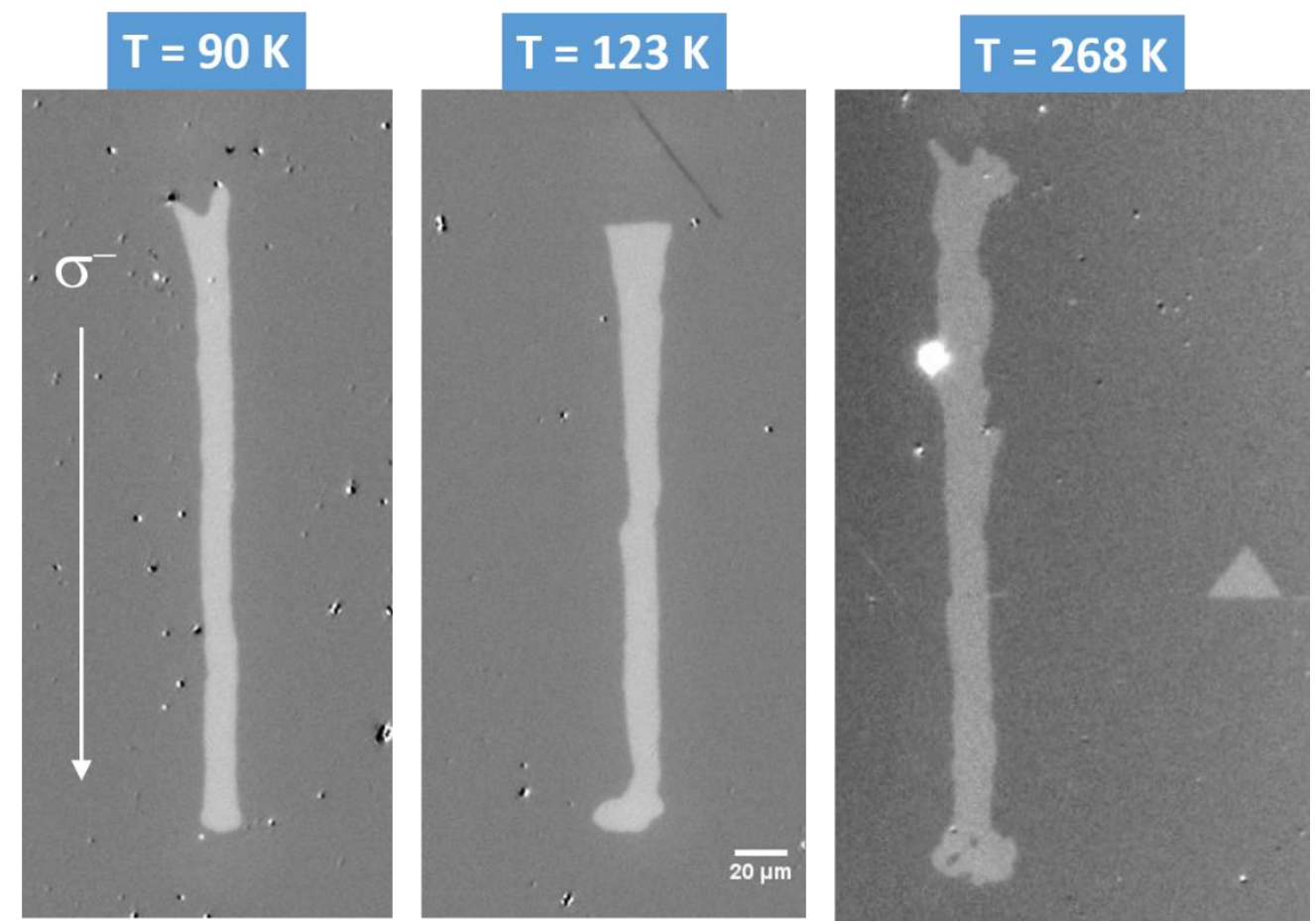

Fig. S17. Demonstration of AOS at different temperatures for sample $F$ $\left[\mathrm{Si} / \mathrm{SiO} 2 / \mathrm{Ta} / \mathrm{Ni}_{3} \mathrm{Pt}(10) / \mathrm{Ir}(0.5) / \mathrm{Co}(1) ; T_{\mathrm{M}}=295 \mathrm{~K}\right]$. The beam was swept from the top to the bottom. The AOS can be observed far below $T_{\mathrm{M}}=295 \mathrm{~K}$ (down to $90 \mathrm{~K}$ ). The optical pump parameters were: $P D=1 \mathrm{ps}, \sigma^{-}$polarization, $100 \mathrm{kHz}$ repetition rate, $F=11 \mathrm{~mJ} / \mathrm{cm}^{2}$. The initial state was set to the APremanent state before every sweep. The triangular domain which can be seen at $T=268 \mathrm{~K}$ has the oppositely oriented remanent state AP+.

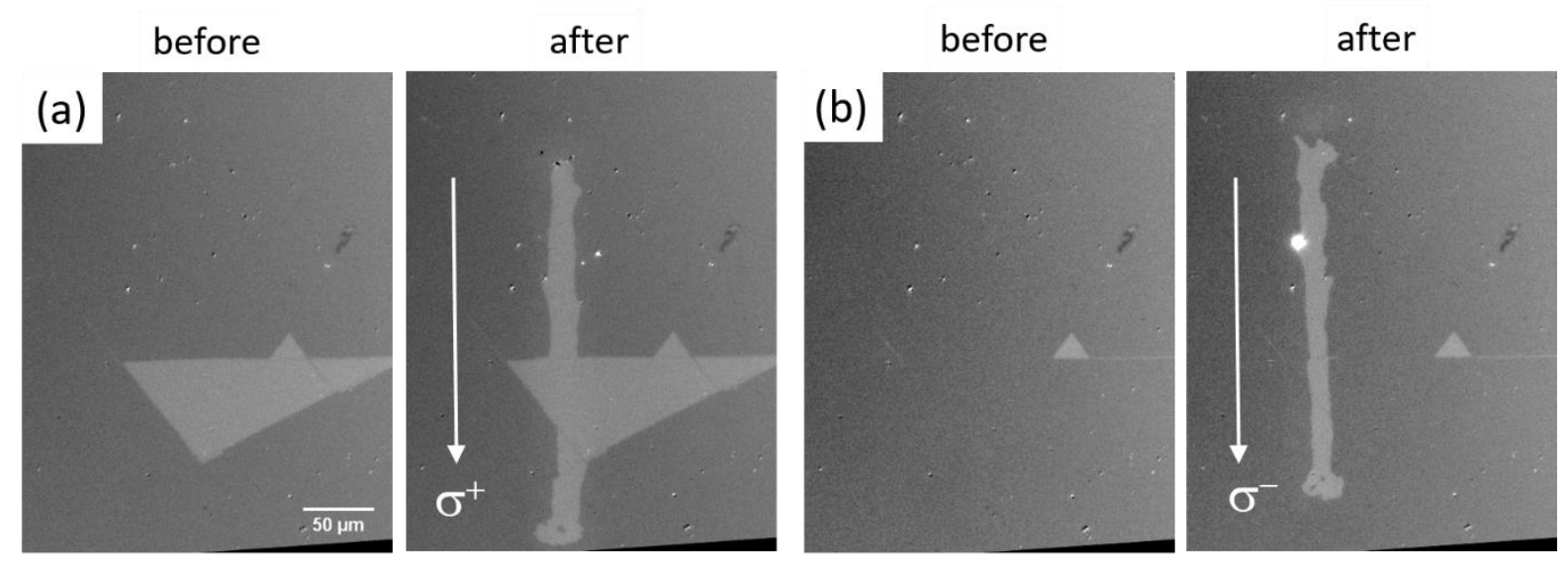

Fig. S18. Demonstration of AOS for circular polarizations (a) $\sigma^{+}$and (b) $\sigma^{-}$. The optical pump parameters were: $P D=1 \mathrm{ps}, 100 \mathrm{kHz}$ repetition rate, $F=11 \mathrm{~mJ} / \mathrm{cm}^{2}$. The images were acquired at $T=$ $268 \mathrm{~K}$ from sample $\mathrm{F}$. The initial state was set to the AP- remanent state before every sweep. 
(a)

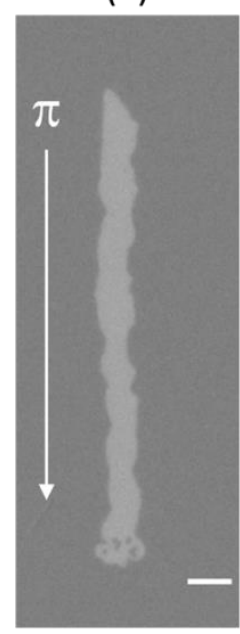

(b)

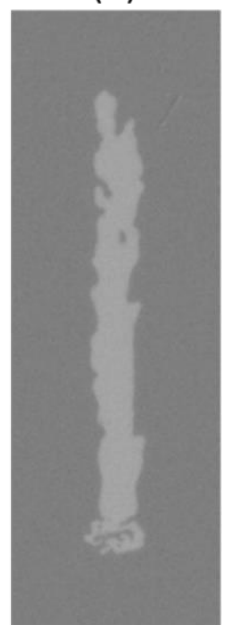

(c)

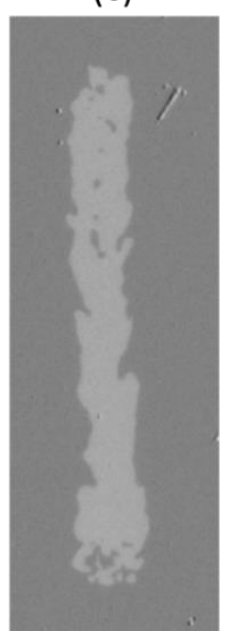

(d)

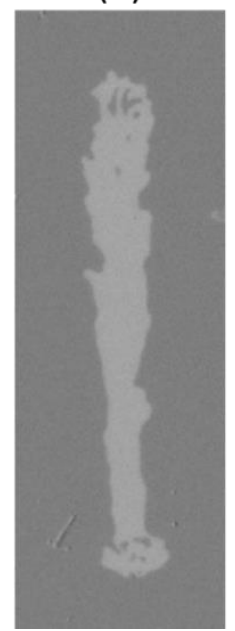

(e)

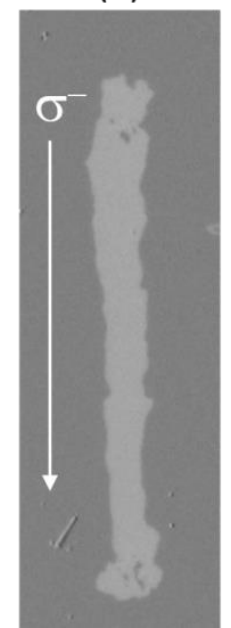

(f)

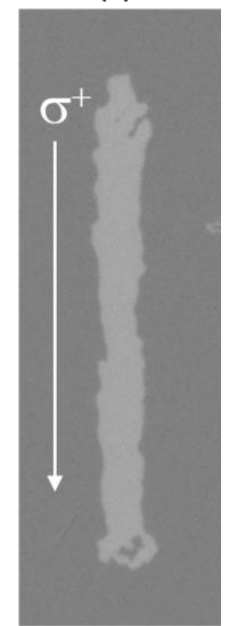

Fig. S19. Demonstration of helicity independent AOS for different polarizations. AOS with linear $p$ pol light $\pi$ for laser fluence $F$ : (a) $6.3 \mathrm{~mJ} / \mathrm{cm}^{2}$; (b) $7 \mathrm{~mJ} / \mathrm{cm}^{2}$; (c) $8.2 \mathrm{~mJ} / \mathrm{cm}^{2}$; (d) $9 \mathrm{~mJ} / \mathrm{cm}^{2}$. AOS with $\sigma^{-}(\mathrm{e})$ and $\sigma^{+}(\mathrm{f})$ circular polarization at $F=11 \mathrm{~mJ} / \mathrm{cm}^{2}$. The optical pump parameters were: $P D=1 \mathrm{ps}$, $10 \mathrm{kHz}$ repetition rate. The images were acquired at $T=260 \mathrm{~K}$ from sample $D$. The initial state was set to the AP- remanent state before every sweep. The scale bar has length of $20 \mu \mathrm{m}$.

(a)

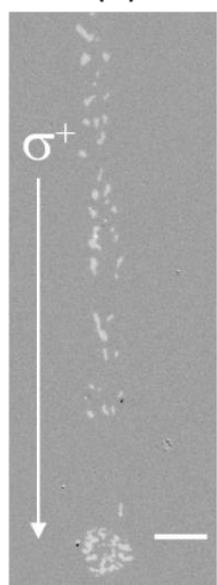

(b)

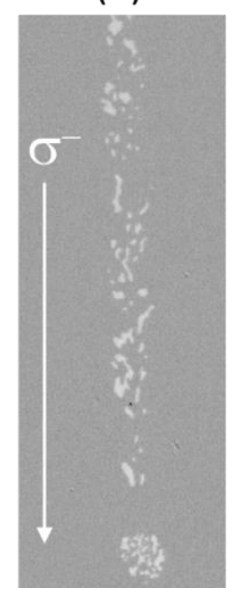

(c)

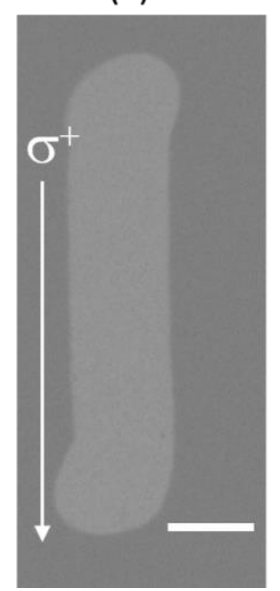

(d)

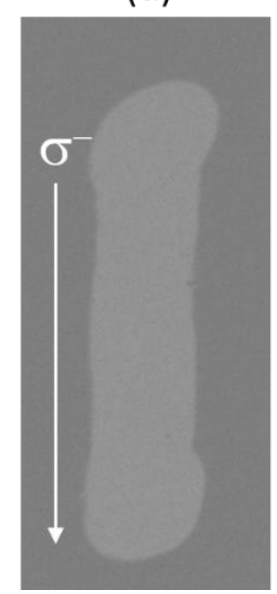

(e)

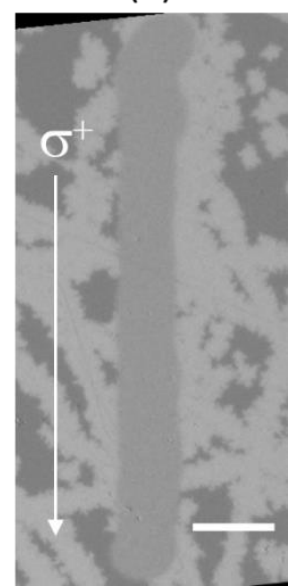

Fig. S20. Demagnetization and optically induced domain creation for reference ferromagnetic samples: (a),(b) - $\mathrm{Si} / \mathrm{SiO}_{2} / \mathrm{Ta}(5 \mathrm{~nm}) / \mathrm{Ir}(3 \mathrm{~nm}) / \mathrm{Co}(\mathbf{1 n m}) / \mathrm{Ir}(3 \mathrm{~nm}) / \mathrm{Ta}(4 \mathrm{~nm}) ; \quad$ (c)-(e) $\mathrm{Si} / \mathrm{SiO}_{2} / \mathrm{Ta}(5 \mathrm{~nm}) /$ $\operatorname{Ir}(3 \mathrm{~nm}) / \mathrm{Ni}_{3} \mathrm{Pt}(\mathbf{1 1 n m}) / \mathrm{Ir}(3 \mathrm{~nm}) / \mathrm{Ta}(4 \mathrm{~nm})$. For the Co film $(\mathrm{a}, \mathrm{b})$, partial switching/domain creation can be observed, with reversed domains being fully switched between the magnetization in the 'down' $\left(M^{-}\right)$and 'up' $\left(M^{+}\right)$direction. The images were obtained with $F=11 \mathrm{~mJ} / \mathrm{cm}^{2}, P D=280 \mathrm{fs}$ and $10 \mathrm{kHz}$ repetition rate. For the $\mathrm{Ni}_{3} \mathrm{Pt}$ film (c,d), demagnetized domains are too small to be resolved by the microscope. Note that even though the optically induced areas appear to be uniform, the average signal is equal to zero, i.e., (the population of $\mathrm{M}^{-}$and $\mathrm{M}^{+}$domain states is equal). The images were obtained with $F=3 \mathrm{~mJ} / \mathrm{cm}^{2}, P D=280 \mathrm{fs}$ and $10 \mathrm{kHz}$ repetition rate. The initial state in (a)-(d) was set to the $M^{-}$remanent state before every sweep. The intensity of the demagnetized region can be easily compared to fully reversed $M^{-}$and $M^{+}$domains by looking at the image obtained by sweeping the beam across the remanent domain structure (e) (obtained with $F=1.8 \mathrm{~mJ} / \mathrm{cm}^{2}, P D=2000 \mathrm{fs}$ and $10 \mathrm{kHz}$ repetition rate). Regardless of the polarization and optical pump parameters, we could not observe AOS for the reference samples. The images were acquired at $T=293 \mathrm{~K}$. The scale bar has length of $50 \mu \mathrm{m}$. 


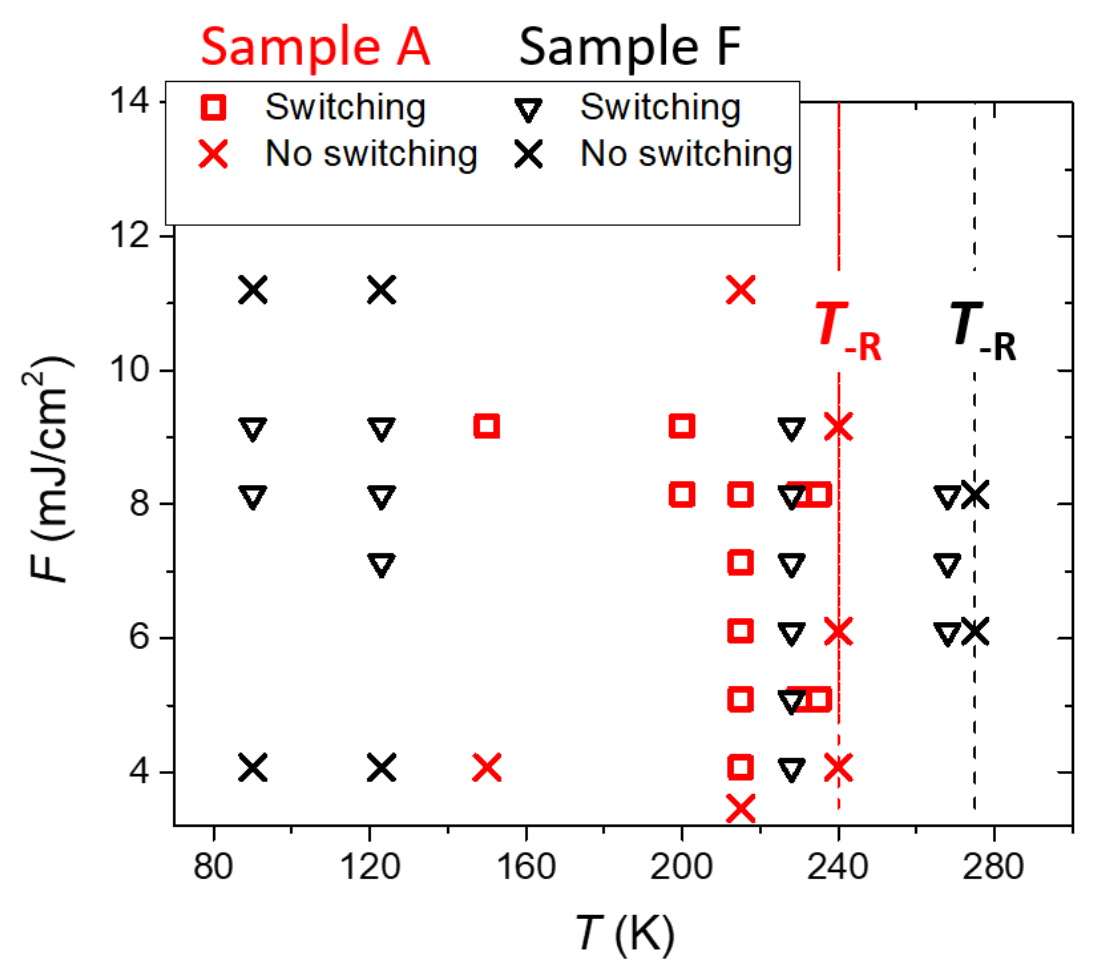

Fig. S21. Diagram showing the switching behavior as a function of sample temperature $T$ and fluence $F$ for sample $A\left[\mathrm{Ni}_{3} \mathrm{Pt}(8.5) / \operatorname{Ir}(0.5) / \mathrm{Co}(1)\right]$ and sample $\mathrm{F}\left[\mathrm{Ni}_{3} \mathrm{Pt}(10) / \operatorname{Ir}(0.5) / \mathrm{Co}(1)\right]$. The optical pump parameters were: $\sigma^{-}$polarization, $P D=1 \mathrm{ps}, 10 \mathrm{kHz}$ repetition rate. For both samples, the AOS can be observed only for temperatures below the transition to negative remanence $T_{-\mathrm{R}}$.

\section{S6. TRMOKE and transfer of spin angular momentum}

Here we discuss the effect of spin angular momentum transfer between the $\mathrm{Ni}_{3} \mathrm{Pt}$ and $\mathrm{Co}$ layers and present complementary time-resolved magneto-optical Kerr effect (TRMOKE) results. The key ingredient for AOS mediated by spin current is a difference in the demagnetization time $\tau_{M}$ of the two layers/sublattices $[2,3,4]$. As a rough approximation $\tau_{M} \sim \mu_{a t} / \alpha$, where $\mu_{a t}$ is the atomic magnetic moment and $\alpha$ is the damping $[2,5,6]$. Here the magnetic moments are 0.3-0.35 $\mu_{\mathrm{B}}$ and $1.687 \mu_{\mathrm{B}}$ for $\mathrm{Ni}_{3} \mathrm{Pt}$ and $\mathrm{Co}$, respectively, as extracted from SQUID measurements (see Fig. S1 in Supplementary Note 1). In addition, it is expected that $\mathrm{Ni}_{3} \mathrm{Pt}$ should have distinctly larger damping since $\mathrm{Pt}$ is known to increase damping in Pt-based alloys due to its large (up to around $0.5 \mathrm{eV}$ ) spin-orbit interaction [7]. Hence, the $\mathrm{Ni}_{3} \mathrm{Pt}$ layer, having both significantly smaller magnetic moment and larger damping, should have a shorter $\tau_{M}$ than the Co layer. However, as shown in Fig. $4 \mathrm{~b}$ in the main text, we see no obvious indication of different $\tau_{M}$ for the two layers in the saturated $\mathrm{P}$ state. This is consistent with TRMOKE measurements performed on separate $\mathrm{Ni}_{3} \mathrm{Pt}$ and $\mathrm{Co}$ reference samples, where similar $\tau_{M}$ values were observed in both cases (see Fig. S24). However, as shown in the main text, there is evidence of different $\tau_{M}$ in the two layers while in the AP state. This can be explained by the presence of spin angular momentum transfer between the layers, which should occur only in the AP state [8]. Our interpretation of the TRMOKE signal has been further verified by additional measurements on sample $E$, which 
has a slightly thinner $\mathrm{Ni}_{3} \mathrm{Pt}$ layer (10 nm instead of $11 \mathrm{~nm}$, for sample $\mathrm{E}$ in Fig. 4), and hence effectively a larger Kerr signal from the Co layer. For this sample, the second local extremum in the TRMOKE signal is correspondingly larger, confirming the assumption of slower demagnetization of the Co layer (see Fig. S22).

Based on the relation $\tau_{M} \sim \mu_{a t} / \alpha$ one could expect a more pronounced difference between the demagnetization times of $\mathrm{Ni}_{3} \mathrm{Pt}$ and Co reference layers, which should be noticeable in the saturated/parallel state and reference samples. Note however that $\tau_{M}$ is also affected by other factors, such as the ambient temperature $T_{0}$ with respect to the Curie temperature $T_{C}$, and it is expected that the relatively high value of $T_{0} / T_{C}$ (within the range of 0.6-0.9 for $\mathrm{Ni}_{3} \mathrm{Pt}$ ) will most likely lead to an increase of $\tau_{M}$ for $\mathrm{Ni}_{3} \mathrm{Pt}$, so that the demagnetization rates of the two layers can be comparable (unless in the presence of spin angular momentum transfer) [6].
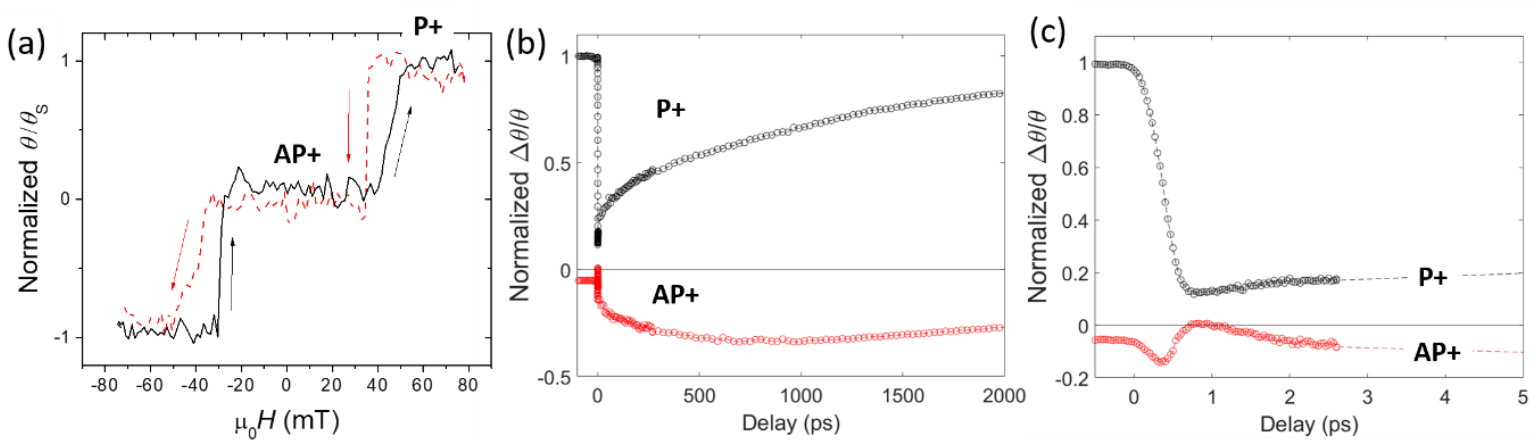

Fig. S22. TRMOKE measurement for sample $D\left[\mathrm{Ni}_{3} \mathrm{Pt}(10) / \mathrm{Ir}(0.5) / \mathrm{Co}(1)\right]$. (a) Kerr hysteresis loop obtained with $400 \mathrm{~nm}$ laser probe. (b) TRMOKE signal $\Delta \theta / \theta$ as a function of time delay for the parallel $(\mathrm{P}+)$ and antiparallel $(\mathrm{AP}+)$ state for fluence $F=6.5 \mathrm{~mJ} / \mathrm{cm}^{2}$. (c) Expanded view of (b) for ultrashort timescales. The size of the TRMOKE signal $\Delta \theta / \theta$ is normalized to the height of the static Kerr hysteresis loop $\theta / \theta_{s}$ measured with the same laser probe as shown in a, so that the size of the optically induced magnetization change can be estimated.

A more detailed explanation of the transfer of angular momentum between layers will require element-specific probes, as have been applied to the GdFeCo alloy [9]. We expect that the $\mathrm{Ni}_{3} \mathrm{Pt}$ will begin to remagnetize in the direction opposite to its initial state, parallel to the Co, for time delays $\tau_{d}$ in the range of $500-1000 \mathrm{fs}$, i.e. as $T$ falls below $T_{\mathrm{C}}$ in the $\mathrm{Ni}_{3} \mathrm{Pt}$ and while the spin current from the Co layer is still present. It is more difficult to say when and how the Co magnetization reorients from the transient $P$ state to the final AP state. Since we cannot reset the initial state after each laser pulse in the TRMOKE experiment, we cannot measure such a reorientation directly. However, we can still observe random switching of the AP state at different delay times as shown in Fig. S23. 

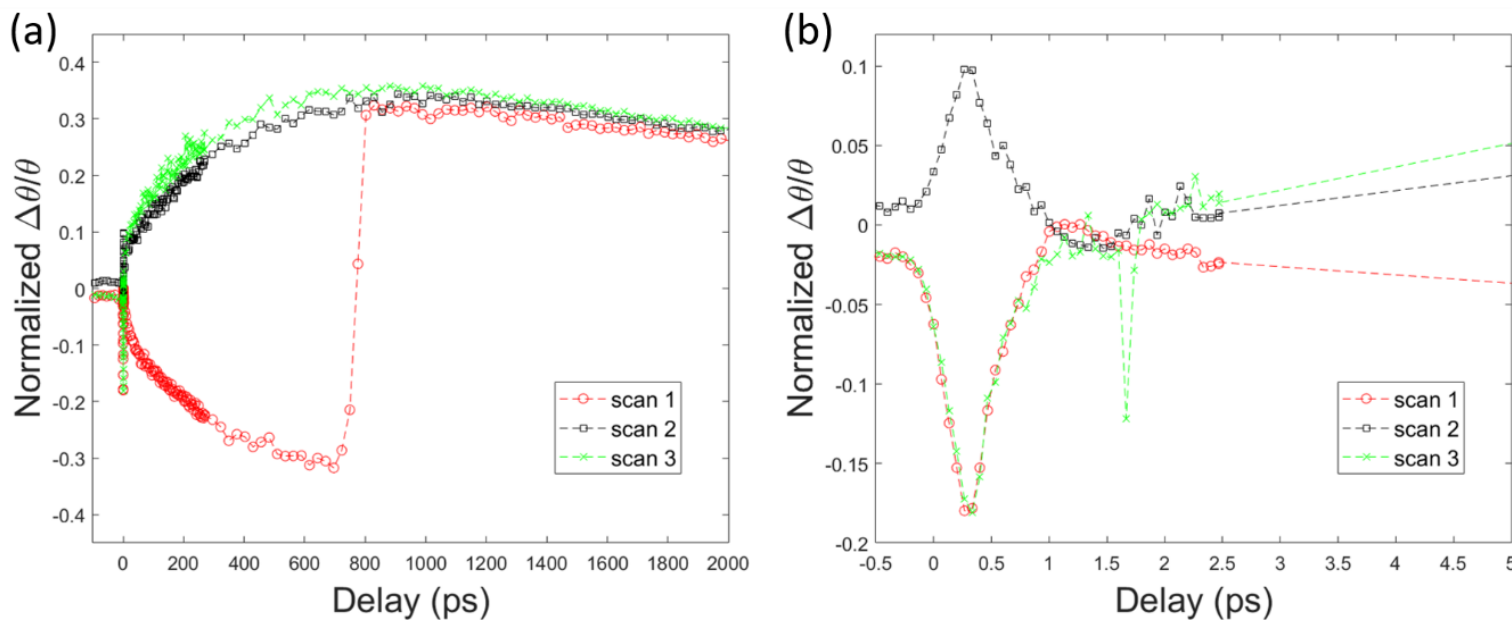

Fig. S23. TRMOKE measurement for sample E $\left[\mathrm{Ni}_{3} \mathrm{Pt}(11) / \operatorname{Ir}(0.5) / \mathrm{Co}(1)\right]$. Subsequent TRMOKE scans show random switching events. For scan 1 , starting from the AP+ initial state, switching manifests as the large change in the TRMOKE signal $\Delta \theta / \theta$ within the $650-750$ ps timescale. Subsequent scan 2, performed without resetting of the initial state, shows the reversed sign of the initial demagnetization peak at around $330 \mathrm{fs}$, which confirms the reversal of the remanent state. For subsequent scan 3 , acquired after resetting the initial state AP+, switching manifests as the change of sign of the TRMOKE signal somewhere between $1.5-2$ ps. (b) expanded view of (a) for shorter timescales.

(a)

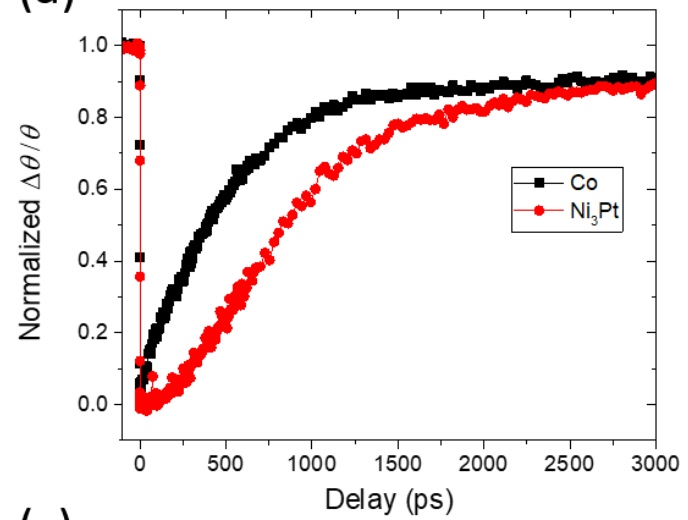

(c)

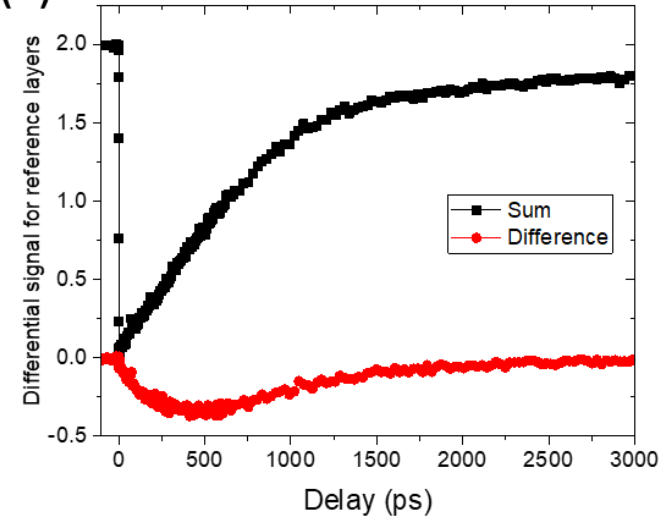

(b)

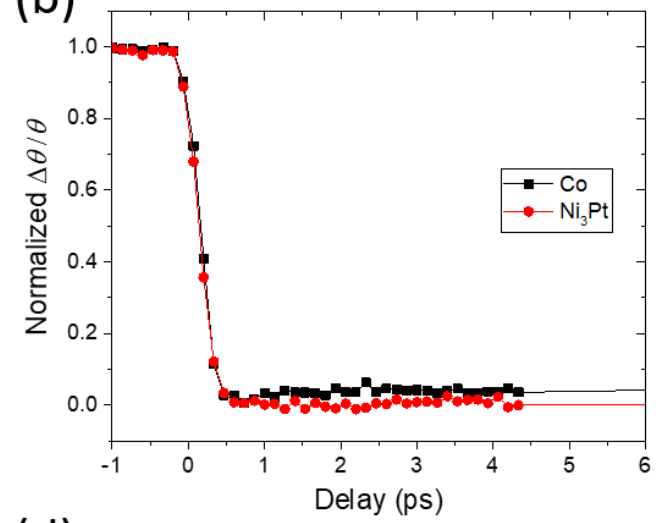

(d)

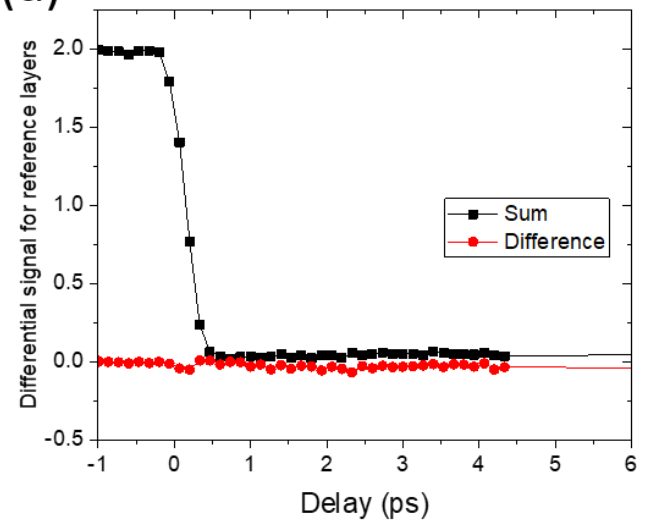

Fig. S24. TRMOKE measurement of the $\mathrm{Ni}_{3} \mathrm{Pt}(11)$ and $\mathrm{Co}(1)$ reference samples. TRMOKE scans (with the Co signal normalized to $\mathrm{Ni}_{3} \mathrm{Pt}$ signal) for (a) longer and (b) shorter time scales. The sum and difference of the TRMOKE signals from the reference samples are shown for (c) longer and (d) shorter time scale. 


\section{References:}

[1] Scott, J. A combined experimental and computational study of $\mathrm{Ni}_{3} \mathrm{Pt} / \mathrm{Ir} / \mathrm{Co}$ synthetic ferrimagnets: Taking atomistic spin simulation into the laboratory. PhD thesis (Queen's University Belfast) (2020).

[2] Evans, R. F. L., Ostler, T. A., Chantrell, R. W., Radu, I. \& Rasing, T. Ultrafast thermally induced magnetic switching in synthetic ferrimagnets. Appl. Phys. Lett. 104, 082410 (2014).

[3] Choi, G.-M. \& Min, B.-C. Laser-driven spin generation in the conduction bands of ferrimagnetic metals. Phys. Rev. B 97, 014410 (2018).

[4] Choi, G.-M., Min, B.-C., Lee, K.-J. \& Cahill, D. G. Spin current generated by thermally driven ultrafast demagnetization. Nature Communications 5, 4334 (2014).

[5] Koopmans, B., Ruigrok, J. J. M., Longa, F. D.\& de Jonge, W. J. M. Unifying ultrafast magnetization dynamics. Phys. Rev. Lett. 95, 267207 (2005).

[6] Koopmans, B. et al. Explaining the paradoxical diversity of ultrafast laser-induced demagnetization. Nat Mater. 9, 259265 (2010).

[7] He, P. et al. Quadratic scaling of intrinsic Gilbert damping with spin-orbital coupling in $L 1_{0}$ FePdPt films: Experiments and $a b$ initio calculations. Phys. Rev. Lett. 110, 077203 (2013).

[8] Malinowski, G. et al. Control of speed and efficiency of ultrafast demagnetization by direct transfer of spin angular momentum. Nature Physics 4, 855 (2008).

[9] Radu, I. et al. Transient ferromagnetic-like state mediating ultrafast reversal of antiferromagnetically coupled spins. Nature 472, 205 (2011). 